\title{
On the 2D nature of flow dynamics in opposed jets mixers
}

DOI:

10.1002/aic. 15566

\section{Document Version}

Accepted author manuscript

Link to publication record in Manchester Research Explorer

\section{Citation for published version (APA):}

Goncalves, N. D., Salvador, H. M., Fonte, C. P., Dias, M. M., Lopes, J. C. B., \& Santos, R. J. (2016). On the 2D nature of flow dynamics in opposed jets mixers. AlChE Journal, 63(6). https://doi.org/10.1002/aic.15566

\section{Published in:}

AIChE Journal

\section{Citing this paper}

Please note that where the full-text provided on Manchester Research Explorer is the Author Accepted Manuscript or Proof version this may differ from the final Published version. If citing, it is advised that you check and use the publisher's definitive version.

\section{General rights}

Copyright and moral rights for the publications made accessible in the Research Explorer are retained by the authors and/or other copyright owners and it is a condition of accessing publications that users recognise and abide by the legal requirements associated with these rights.

\section{Takedown policy}

If you believe that this document breaches copyright please refer to the University of Manchester's Takedown Procedures [http://man.ac.uk/04Y6Bo] or contact uml.scholarlycommunications@manchester.ac.uk providing relevant details, so we can investigate your claim.

\section{OPEN ACCESS}




\title{
ON THE 2D NATURE OF FLOW DYNAMICS IN OPPOSED JETS
}

\section{MIXERS}

\author{
N. D. Gonçalves ${ }^{\text {a }}$, H. M. Salvador a, C. P. Fonte ${ }^{\text {b }}$, M. M. Dias ${ }^{\text {a }}$, J. C. B. Lopes ${ }^{\text {a }}$, R. J. \\ Santos $^{\mathrm{a} *}$
}

${ }^{a}$ Laboratory of Separation and Reaction Engineering - Laboratory of Catalysis and Materials (LSRE-LCM), Universidade do Porto, Faculdade de Engenharia, Rua Dr. Roberto Frias, 4200-465 Porto, Portugal; e-mail: rsantos@fe.up.pt

${ }^{b}$ School of Chemical Engineering and Analytical Science, The University of Manchester, Oxford Road, Manchester M13 9PL, United Kingdom.

\begin{abstract}
Confined impinging jets (CIJs) are reactors used in processes that require fast mixing. In such equipment two fluids are injected from opposite sides of a chamber, impinging into each other and forming flow structures that enable an effective mixing and reaction. The turbulence analysis shows that the energy is injected from smaller scales, having approximately the injectors width, that feed larger scale structures up to larger vortices that occupy the entire mixing chamber width. This energy distribution has an inverse energy cascade, i. e. it is an inversion of the traditional description of homogeneous 3D turbulence. The typical flow scales of 2D CIJs are clearly shown in this work to be linked to the $2 \mathrm{D}$ turbulence energy spectrum and to integral scales of turbulence. Moreover, the turbulence mechanisms in 3D CIJs at transitional flow regimes are shown to be similar to 2D CIJs. This is to our knowledge the first demonstration of 2D turbulence in an industrial mixer/reactor.
\end{abstract}

Keywords: Confined Impinging Jets (CIJs), 2D turbulence, mixing, inverse energy cascade, Computational Fluid Dynamics (CFD). 


\section{INTRODUCTION}

\section{Inverse energy cascade}

2D turbulence is characterized by an inverse energy cascade where the energy is injected from the small scales that will feed larger eddies ${ }^{1}$. This is an inversion of the traditional description of homogeneous turbulence, in $3 \mathrm{D}$, where the energy of the large scales is transferred to smaller scales down to the viscous dissipation ${ }^{2}$. In $3 \mathrm{D}$ turbulent flows there is vortex stretching, whereas in 2D turbulent flows the freedom of movement in a third dimension of the flow is constrained, which prevents this mechanism. This leads to the scenario described by Kraichnan ${ }^{3}$ of the conservation of enstrophy (mean squared vorticity) and the existence of two energy cascades: an inverse energy cascade where energy is transferred to larger lengthscales, and an enstrophy cascade where the energy is transferred to smaller lengthscales, both cascades have the energy injection at the same lengthscale, $\lambda_{i n j}^{-1}{ }^{4}$.

Whereas the inverse energy cascade dominates the flow for the scales larger than $\lambda_{\text {inj }}^{-1}$, for smaller scales the enstrophy cascade dominates the flow ${ }^{1,4}$. Batchelor ${ }^{5}$ predicted the existence of a cascade of mean-square vorticity at large Reynolds number and an equilibrium range in the vorticity spectrum. Later, Amarouchene and Kellay ${ }^{6}$ confirmed Batchelor's theory with experimental work, indicating an exponent of $-5 / 3$ for the scaling law of the power spectrum in the inertial subrange, when the scalar field is affected by both inverse energy and enstrophy cascades, and an exponent of -1 when the passive scalar field is affected only by the enstrophy cascade.

Up to date, the studied turbulent flows with $2 \mathrm{D}$ behaviour enabled to build an already considerable body of theory ${ }^{1}$, although in most of the cases these flows have been too conceptual as far as Chemical Engineering practice is regarded. The two most studied cases are the flowing soap films ${ }^{7-14}$ and the electromagnetic layers ${ }^{7,13,15-26}$. These flows have the 
turbulence restricted to $2 \mathrm{D}$ because they are restricted to a thin layer that prevents vortices to stretch in the normal direction to the flow. A 2D behaviour can also stem from additives, such as macromolecules that align with flow direction and lend it a strong dimensionality ${ }^{4}$, or from the forcing of an inlet boundary condition that imposes a markedly $2 \mathrm{D}$ behaviour to the flow. Such is the case of small scale mixers with opposed jets, namely those where the inlet boundary condition extends throughout the entire reactor depth.

\section{CIJs}

Confined Impinging Jets (CIJs) are highly efficient mixing devices composed of a confining chamber, two opposed injectors near the closed top of the chamber and an outlet at the open end from where the fluid leaves the reactor ${ }^{27}$. The flow in opposed jets is mainly affected by the dynamics in the plane of the jets, a plane defined by jets' axis and mixing chamber's axis. As a consequence, the flow in CIJs has been extensively studied from 2D physical models that were validated and compared with actual 3D physics in previous works of Santos et al. ${ }^{28,}$ 29.

The first opposed-jets mixers were cylindrical with two opposed round jets ${ }^{30}$ a geometry typically referred to as Confined Impinging Jets mixers/reactors. The first studies on CIJs were focused in the mixing process for Reaction Injection Moulding (RIM) processes, where CIJs typically have chamber diameter $1 \mathrm{~cm}$ and diameter of the injectors from 1 to $3 \mathrm{~mm}$. Malguarnera and Suh ${ }^{30}$ studied the effect of the Reynolds number and the momentum ratio of the opposed jets on the flow, and they proposed that two conditions to obtain a better mixing of the two monomers (isocyanate and polyol) are: the Reynolds number should be larger than 50 and the momentum of the opposed jets should be equal. Other authors have reached similar conclusions on the effect of the Reynolds and the jets' momentum ratio effect on the flow ${ }^{27,31-43}$. The abrupt increase of mixing quality is due to a flow regime transition, which is mainly based on the jets Reynolds number, and the jets impose a strong dimensionality to the 
flow in CIJs, making the strongest flow dynamics to occur mainly in the plane defined by the mixing chamber axis and the jets axis.

Later, with the advent of micro reactors, prismatic geometries of opposed jets reactors with opposed round or rectangular injectors became common ${ }^{44-48}$. These geometries are referred to as $\mathrm{T}$-jets reactors and can be regarded as passive lamination mixers when operating at low Reynolds numbers ${ }^{49}$, or chaotic mixers for larger Reynolds numbers. Since in most T-jets the inflow extends through all chamber depth, there is a strong effect of dimensionality that lends these mixers dynamics a markedly 2D behaviour.

In this paper, flow data for opposed jets mixer is obtained from 2D and 3D simulations, which results are analysed within the framework of the statistical description of $2 \mathrm{D}$ turbulence. Hydrodynamic lengthscales from turbulence energy spectra are compared with the actual 3D flow and are shown to be set from the CIJs dimensions.

\section{PROBLEM DESCRIPTION}

\section{Geometry}

Opposed Jets Reactors consist of rectangular (2D case - see Fig. 1a), prismatic or cylindrical (3D case - see Fig. 1b) mixing chambers with two directly opposite injectors, which are placed near the closed top of the chamber. The chamber has an open bottom from where the fluid leaves. The chamber considered in this work has a diameter or width, depending if it is $3 \mathrm{D}$ or $2 \mathrm{D}$, of $D=10 \times 10^{-3} \mathrm{~m}$ and a height $H=5 D=50 \times 10^{-3} \mathrm{~m}$. The injectors have a diameter or width $d=1.5 \times 10^{-3} \mathrm{~m}$ and are placed at $D / 2$ from the chamber top. The origin of the coordinate axis is defined at the intersection of the injectors axes and the mixing chamber axis: this point is defined as the impact point. The contact or impingement point is the actual place where the opposed jets contact, and due to the jets bending in $2 \mathrm{D}$ geometries this point is displaced to a distance $d$ from the axis of the injectors towards the outlet. The 
placement of the contact point can be seen in Fig. 2a with the streamlines and vorticity sign for the $2 \mathrm{D}$ case, and in Fig. $2 \mathrm{~b}$ from the vorticity sign for the 3D case.

\section{Boundary Conditions}

At the injectors, it was considered a parabolic velocity profile, that occurs in fully developed velocity profiles in rectangular (2D case) and cylindrical (3D case) domains, according to

$$
\begin{aligned}
& v(x)=\frac{3}{2} v_{\text {inj }}\left(1-\left(\frac{x}{d / 2}\right)^{2}\right) \\
& v(r)=2 v_{\text {inj }}\left(1-\left(\frac{r}{d / 2}\right)^{2}\right),
\end{aligned}
$$

respectively for the $2 \mathrm{D}$ and $3 \mathrm{D}$ cases, having at the inlet jets an average velocity $v_{\text {inj }}$. A constant and uniform pressure was imposed at the outlet boundary, and at walls (chamber top and sides) it was considered the no-slip boundary condition.

The fluid density is $\rho=10^{3} \mathrm{~kg} / \mathrm{m}^{3}$ and the dynamic viscosity was set $\mu=0.02 \mathrm{~Pa} \cdot \mathrm{s}$, in order to have a Reynolds number $R e=\rho v_{i n j} d / \mu=300$. This viscosity value is representative of some RIM formulations, although in the lower end of the viscosity range used in industry. Furthermore, there is a considerable body of experimental data using these fluids from previous works $27,29,40,42,43,50$.

The initial condition, was a symmetric and steady state solution, obtained with a no shear wall set at the symmetry plane/axis, for $2 \mathrm{D} / 3 \mathrm{D}$ case respectively.

\section{CFD model}

The 2D domain (Fig. 1a) was discretized with a structured and orthogonal mesh of quadrilateral elements, which maximum edge length is $50 \times 10^{-6} \mathrm{~m}$, leading to a total number of $2 \times 10^{5}$ elements. The 3D domain (Fig. 1b) was discretized with a unstructured mesh, which maximum edge length is $100 \times 10^{-6} \mathrm{~m}$, with a total number of $2 \times 10^{6}$ elements. 
The commercial finite volume CFD software ANSYS Fluent ${ }^{\mathrm{TM}}$ was used to solve the transient pressure-velocity problem modelled by the continuity (mass conservation) equation

$$
\nabla \cdot \boldsymbol{u}=0
$$

and the momentum conservation equation

$$
\rho\left(\frac{\partial \boldsymbol{u}}{\partial \mathrm{t}}+\boldsymbol{u} \cdot \nabla \boldsymbol{u}\right)=-\nabla p+\mu \nabla^{2} \boldsymbol{u}
$$

The pressure-velocity coupling was done with the COUPLED scheme. The PRESTO! scheme has been chosen to discretize the pressure gradient terms, and the Second Order Upwind scheme to discretize the advective terms of Eq. 4 . The convergence criterion for the maximum normalized residuals of continuity and momentum conservation equations is $10^{-4}$.

During a period equal to the mean residence time of the fluid in the mixing chamber, $t=\tau$, the flow evolves from the steady state to a fully developed chaotic flow, and so these data were ignored. The turbulence analysis is made for a flow time equal to $15 \tau$.

The time step for the unsteady flow simulations was defined from the Courant number, $C=$ $v_{\max } \delta_{t} / \delta_{x} \leq 1$, assuming the maximum velocity at the injectors centre and the elements size as the reference length. The velocity at injectors centre, is $v_{\max }=1.5 v_{i n j}=6 \mathrm{~m} / \mathrm{s}$ for the $2 \mathrm{D}$ case and $v_{\max }=2 v_{\text {inj }}=4 \mathrm{~m} / \mathrm{s}$ for the $3 \mathrm{D}$ case, and so $\delta_{t} \leq \frac{25}{3} \times 10^{-6} \mathrm{~s}$ and $\delta_{t} \leq 2.5 \times 10^{-5} \mathrm{~s}$, for $2 \mathrm{D}$ and $3 \mathrm{D}$ simulations, respectively. The $2 \mathrm{D}$ time step was set to $8.333 \times 10^{-6} \mathrm{~s}$ whereas for the $3 \mathrm{D}$ case the time step is $2.5 \times 10^{-5} \mathrm{~s}$.

The kinetic energy injected into the system is

$$
\left\langle\kappa_{i n j}\right\rangle=\frac{1}{d} 2 \int_{-d / 2}^{d / 2} \frac{1}{2} v^{2} d x=\frac{6}{5} v_{i n j}^{2}
$$

and the turbulent kinetic energy at each point is defined as

$$
\kappa=\frac{1}{2}\left(u^{\prime 2}+v^{\prime 2}\right)
$$

where $u^{\prime}$ and $v^{\prime}$ are the fluctuating velocity terms, $u^{\prime}=u-\bar{u}$ and $v^{\prime}=v-\bar{v}$. The results of the turbulent kinetic energy are normalized as $\kappa^{*}=\kappa /\left\langle\kappa_{i n j}\right\rangle$, and the time averaged value, $\overline{\kappa^{*}}$, 
is computed over 1000 evenly sampled time steps from the simulated 15 mean residence times.

The energy dissipation rate is also calculated at each time step as

$$
\varepsilon=2 v\left(s_{i j} \cdot s_{i j}\right)
$$

where $s_{i j}=\frac{1}{2}\left(\frac{\partial u_{i}^{\prime}}{\partial x_{j}}+\frac{\partial u_{j}^{\prime}}{\partial x_{i}}\right)$ is the fluctuating rate of strain tensor, and $v=\mu / \rho$ is the kinematic viscosity. The energy dissipation rate results are time averaged over the 1000 sampled time steps. The sampling period for this data is $15 \tau / 1000$, which corresponds to a sampling frequency of $f=1600 \mathrm{~Hz}$. The flow frequencies of the jets oscillations and of the vortices passages are around $240 \mathrm{~Hz}\left(f_{\text {vortex }}=\frac{d v_{\text {inj }}}{2 A_{\text {vortex }}}\right.$, with the vortex diameter equal to $\left.\frac{4}{5} \frac{D}{2}\right)$ and $80 \mathrm{~Hz}\left(f_{\text {vortex }}=\frac{d v_{\text {inj }}}{A_{\text {vortex }}}\right.$, with the vortex diameter equal to $\left.D\right)$, thus the sampling is not in phase with the flow and thus the sampling period is not introducing a bias in the results.

\section{RESULTS}

\section{Turbulence dynamics in 2D}

The velocity dynamics at the impact point of an opposed jets mixer has a complex quasioscillatory behaviour that is described with detail in previous works $29,33,36,42$. The flow in opposed jets at chaotic flow regimes, where the Reynolds number is still in the laminar range, has been mainly assessed from the frequencies of flow oscillations $29,33,51-55$. The frequency of the jets oscillations is set from the rate of formation of the vortices ${ }^{29}$ that issue from the jets impingement point creating a vortex street throughout the mixing chamber. Fig. 3 shows a short sequence of flow maps from 2D simulation over a period of $0.24 \tau$, which is long enough to show the evolution of a vortex over a distance larger than one chamber width $(>D)$. From the flow maps sequence it is seen the vortices moving towards the outlet maintaining approximately their shape, which only evolves due to wall shearing and from interaction with 
neighbouring vortices. Immediately bellow the inlet jets a pair of vortices is formed, one vortex on each side of the chamber, that will evolve into vortices having diameter $D$. The formation of these vortices and detachment towards the outlet is associated with the rate of oscillation of the flow at the opposed jets contact region. Fig. 3 shows an image sequence of the evolution of the jets contacting region with the surrounding vortices, namely the jets contacting angle: the vortices grow and push the contact point and after when the vortices detach the jets are pulled. This dynamics of vortex formation promotes a flapping of the jets in the impingement region.

A similar flow dynamics is observed for 3D geometries, see Fig. 4, where $z$-vorticity isosurface maps give an indication of the sense of rotation of the flow. Two maps covering a period equal to $0.02 \tau$ show the flapping of a pancake like structure, described by Wood et al. ${ }^{33}$, that stems from the jets impingement point. The mechanisms promoting oscillations of the flow in $3 \mathrm{D}$ are also associated with the vortices formation dynamics ${ }^{29}$. The complex patterns that the fluid follows after issuing from the injectors are seen from the streamlines in Fig. 4. The vortices engulf both flow streams, as can be seen from the fact that streamlines from opposite injectors (red and black) end up meandering together throughout the mixing chamber.

The flow dynamics in opposed jets mixers has been described from the typical values of the flow oscillation frequencies $29,33,36,51,53,56-58$. Nevertheless, the velocity dynamics data show far more features than the dominant oscillation frequencies, which are associated with the several flow scales in the mixing chamber. The complete information from the velocity dynamics data is obtained from the power spectrum, which is shown in Fig. 5a for $R e=300$ at the impact point. The power spectrum from the velocity data in Fig. 5a has a large amount of high frequency noise that can difficult its analysis. To ease the analysis of the power spectrum of Fig. 5a, the high frequency noise was eliminated using a Hamming window function, $W$, 


$$
E_{\text {filtered }}\left(\lambda_{i}\right)=\frac{\sum_{j=-\frac{n-1}{2}}^{\frac{n-1}{2}} W(j) E\left(\lambda_{i+j}\right)}{\sum_{j=-\frac{n-1}{2}}^{\frac{n-1}{2}} W(j)}, W(j)=\alpha-\beta \cos \left(\frac{j}{n-1} 2 \pi\right)
$$

where $\lambda_{i}$ is the wavenumber. The filtered power spectrum is shown in Fig. 5b. All power spectra reported hereafter are filtered using the Hamming window and considering $n=41$ and $\alpha=-\beta=1 / 2$.

The power spectra of velocity data along the flow is set from the turbulence scales. In the opposed jets chamber the length of the vortex street issuing from the jets impingement point is limited, and so a long simulation period is necessary to generate a data series long enough for the turbulence analysis. From the time series a power spectrum in the frequency domain is obtained. The time scale is converted into a wavelength using a reference velocity equal to the average velocity at the mixing chamber outlet, $v_{\text {out }}=1.2 \mathrm{~m} / \mathrm{s}$, and so the frequency $f$ is converted to wavenumber as $\lambda=f / v_{\text {out }}$.

Vortices with different diameters occur at different locations, leading to different velocities. From $x=d$ to $x=d+D / 2$, vortices with diameter approximately $D / 2-d$ are formed, as can be seen in Fig. $2 \mathrm{a}$ from the vorticity sign. These vortices have an area $A_{\text {vortex }} \approx$ $\pi((D / 2-d) / 2)^{2}$ and are fed by one injector with flow rate $q=d v_{i n j}$, and their formation frequency is $f_{\text {form }} \approx q / A_{\text {vortex }}$. The velocity of these vortices can be approximated from their formation frequency and diameter $v_{\text {vortex }} \approx D_{\text {vortex }} f_{\text {form }} \approx 2.2 \mathrm{~m} / \mathrm{s}$. Near the chamber exit the larger vortices have a diameter similar to the chamber width and area $A_{\text {vortex }} \approx$ $\pi(D / 2)^{2}$. These vortices, are fed by both injectors with flow rate $q=2 d v_{\text {inj }}$, and have formation frequency $f_{\text {form }} \approx q / A_{\text {vortex }}$ yielding a velocity $v_{\text {vortex }} \approx$ $D_{\text {vortex }} f_{\text {form }} \approx 1.5 \mathrm{~m} / \mathrm{s}$.

The power spectra for several points along the chamber and for $\mathrm{Re}=300$ are shown in Fig. 6. The main scales of the flow are marked in each power spectra. The smallest scale is the inlet jets width, $d$, with the corresponding wavenumber $\lambda=1 / d \approx 6.7 \times 10^{2} \mathrm{~m}^{-1}$. This 
injection scale is larger than the Kolmogorov scales, which will be shown to be $l_{K} \approx 75 \mu \mathrm{m}$ for $\operatorname{Re}=300$ in the section on integral scales in this paper. The largest scale is the diameter of the larger vortices that extend throughout the entire chamber width, $D$, and the corresponding wavenumber is $\lambda=1 / D=10^{2} \mathrm{~m}^{-1}$. Vortices with approximately half the diameter of the chamber width, i.e. $D / 2$, occur in a region from the jets contact point, which is approximately at $x=d$, to $x=d+D / 2$. The mixing chamber height, $H$, is also marked in the plots.

The power spectra at the region where the jets impinge $x=d$, to $x=d+D / 2$ have a clear concentration of energy at a scale associated with the vortices having diameter $D / 2-d$, which defines the energy containing range in this flow region. From the large scales to a wave number $\lambda \approx 1.200 \times 10^{3} \mathrm{~m}^{-1}$ the energy transfer between scales is at equilibrium, i.e. $E \propto$ $\lambda^{-5 / 3}$. The energy injection scale is identified by the slope change to a lower value than $-5 / 3$, which occurs at $\lambda \approx 1.200 \times 10^{3} \mathrm{~m}^{-1}$, different from the expected value $d^{-1} \approx$ $6.67 \times 10^{2} \mathrm{~m}^{-1}$. If the wavenumber is obtained considering for the reference velocity the vortices velocity (with diameter $D / 2-d$ ) $v_{\text {vortex }} \approx 2.2 \mathrm{~m} / \mathrm{s}$, the wavenumber value is circa $d^{-1}, \lambda \approx 6.5 \times 10^{2} \mathrm{~m}^{-1}$

The injection scale of energy, $d^{-1} \approx 6.67 \times 10^{2} \mathrm{~m}^{-1}$, is transferred to the larger scale $D / 2$, inverting the turbulent energy cascading, which in this case is transferred from the smaller to the larger lengthscales ${ }^{1,3}$. The energy cascade is not at equilibrium in this region because the relation $E \propto \lambda^{-5 / 3}$ is not verified in the inertial subrange from $\lambda=2 D^{-1}$ to $\lambda=d^{-1}$. The flow at this region is also not isotropic as can be seen from the different energy for the $x$ and $y$ flow directions in the power spectra of Fig. 6.

At $x=d+D / 2$, two vortices with a diameter $D / 2$ evolve and will merge into larger vortices downstream of $x=d+D$. At the point $x=d+D / 2$ there is an equilibrium of the energy transferred from the injection scale, $x=d$, to the energy containing scale, $D / 2$. The equilibrium condition is seen in Fig. 6f with a scaling of $E \propto \lambda^{-5 / 3}$ in the inertial subrange. 
The turbulence at this point is isotropic, the strong dimensional biasing of the flow that is caused by the jets around the impingement is no longer observed at $x>d+D / 2$.

Until $x=d+D / 2$, the energy containing scales are around $D / 2$, which is the diameter of the two vortices that are formed downstream the jets impingement point. These vortices engulf each other evolving to vortices with a diameter that extend throughout the entire mixing chamber width, $D$. The $D / 2$ vortices engulfment occur from $x=d+D / 2$ to $x=d+$ $D$, and in this region the energy containing scales shift from $\lambda=(D / 2)^{-1}$ to $\lambda=(2 D)^{-1}$, as can be seen from Fig. 6f and Fig. 6g. While the vortices with diameter $D / 2$ cause a wavenumber of $(D / 2)^{-1}$, in the vortex street where the vortices diameter is $D$, the wavenumber of the energy containing scale is $(2 D)^{-1}$, i.e. it is given by twice the vortices diameter. In this case, each wavelength is associated to the passage of a pair of counterrotating vortices, and thus $\lambda=(2 D)^{-1}$. In the power spectra are seen local maxima around $\lambda=(D / 2)^{-1}$ and $\lambda=(2 D)^{-1}$, with a decreasing relevance of the $\lambda=(D / 2)^{-1}$ energy peak. This is coherent with the fact that the smaller vortices engulf each other around this region, and at downstream positions only the vortices with diameter $D$ generate observable turbulent dynamics. The energy injection scale is also seen at these downstream positions from the slope inflection in the power spectrum. The energy transfer is now from the small scale $\lambda^{-1}=d$ to the large scale $\lambda^{-1}=2 D$ closely matching the equilibrium condition $E \propto \lambda^{-5 / 3}$.

Near the mixing chamber outlet, the larger vortices occupy the whole chamber width, and have velocity $v_{\text {vortex }} \approx 1.5 \mathrm{~m} / \mathrm{s}$ as referred above. It should be noticed that due to the alternate rotation direction of the vortices the wavenumber measured from velocity data corresponds to a pair of vortices. These vortices with diameter $D$ should be detected by wavenumber $\lambda=(2 D)^{-1}=50 \mathrm{~m}^{-1}$. This value is very close to the local maximum value associated to the energy containing scale in Fig. 6i. The energy injection scale is not identifiable at the locations further downstream in the mixing chamber. 
The strong spatial evolution of the turbulence dynamics shows that in this $2 \mathrm{D}$ mixer the turbulence is not homogenous. In the regions where there is a transition between lengthscales there is anisotropy, while in the regions where the flow scales are not evolving the turbulence is isotropic.

The 2D turbulence dynamics was studied for different Reynolds numbers. Fig. 7 shows the power spectra at point $(0, d+D / 2)$ for Reynolds number 200, 250, 300, 400 and 500. For all the simulated Reynolds numbers the turbulence energy transfer between scales is at equilibrium when $E \propto \lambda^{-5 / 3}$ from $\lambda \approx(D / 2)^{-1}$ to $\lambda \approx(d / 2)^{-1}$. The injection scale is seen from the shift in the power spectra slope from $E \propto \lambda^{-5 / 3}$ to $E \propto \lambda^{-m}$ where $m>5 / 3$. For all the Reynolds numbers the injection of energy is observed circa $\lambda \approx(d / 2)^{-1} \approx 1.333 \times$ $10^{3} \mathrm{~m}^{-1}$. This wavenumber value, obtained by the division of frequency $f$ by $v_{\text {out }}=$ $1.2 \mathrm{~m} / \mathrm{s}$, would be approximately $d^{-1}$ if the reference velocity was the velocity of the vortices that are formed after the injectors, $v_{\text {vortex }} \approx 2.2 \mathrm{~m} / \mathrm{s}$. The energy containing range is also in the same range of wavelengths for all Reynolds numbers, i.e. $\lambda \approx(D / 2)^{-1}$. The main effect of the Reynolds number is the decrease of the local maxima of energy around $\lambda \approx$ $(D / 2)^{-1}$. This decrease is associated to an increase on the range of vortices sizes or an increase on the vortices shape distortion, which makes the energy associated with these vortices not so concentrated and so the energy in these maxima spreads around the $\lambda \approx$ $(D / 2)^{-1}$

The simulated Reynolds numbers swept the range of typical operation regimes for opposed jets reactors, particularly for micro sized T-jets and RIM. In this range the distribution of energy, namely the wavelengths or wavenumber for the inertial sub-range are kept constant, and thus the flow scales do not change within these Reynolds numbers. For fully turbulent flows, the 2.5 fold increase in Reynolds number should promote a decrease in the smaller turbulence scales of approximately twofold, $l_{K} \propto \mathrm{Re}^{-3 / 4}$, where $l_{K}$ is the Kolmogorov 
lengthscale. In this flow the smallest observable scale is defined by the jets and keeps constant in the range $200 \leq \operatorname{Re} \leq 500$.

\section{Turbulent kinetic energy and dissipation in 2D}

The distribution of the normalized turbulent kinetic energy $\overline{\kappa^{*}}$ along the chamber axis was analysed, as well as its evolution with Re. It can be seen in Fig. 8 that the shape of the turbulent kinetic energy distribution is analogous for all the studied cases. In the impact point there is low kinetic energy with an increase until circa $x=d+D / 16$, which is close to the opposed jets contact point. This local maximum is due to the onset of the vortices with $D / 2$ diameter that are being formed immediately downstream of the injectors. Further downstream the kinetic energy diminishes until $x \approx d+D / 4$, probably due to the fact that the vortices with $D / 2$ are formed in pairs, side by side, and thus the flow in the chamber axis is not so affected by the vortices formation dynamics in this region of the mixing chamber. From $x \approx$ $d+D / 4$ to $x \approx d+2 D$ the vorticity increases again due to the growth of the energy containing vortices from a lengthscale of $D / 2$ to $D$. After $x \approx d+2 D$, the turbulent kinetic energy decreases due to viscous dissipation.

The mean value of turbulent kinetic energy dissipation rate, $\bar{\varepsilon}$, is also analysed along the mixing chamber axis. It can be seen in Fig. 9 that the location of the maximum dissipation is similar for all the Re, circa $x=d+D / 8$. This maximum $\bar{\varepsilon}$ is associated to the region of maximum shearing, which is the point where the two opposed jets contact. From this point on, the turbulent kinetic energy dissipation rate decreases towards, the outlet. This decrease is steeper from $x \approx d+D / 4$ to $x \approx d+D$ in a region where the vortices scales are evolving.

From the average values of the turbulent kinetic energy dissipation rate in the flow domain, the Kolmogorov turbulence lengthscale is $l_{k}=\sqrt[4]{v^{3} / \varepsilon} \approx 75 \mu \mathrm{m}$, where $v$ is the kinematic viscosity. This scale is smaller than the flow structures identified from the flow maps, see Figure 2, or from the spectral analysis of 2D turbulence, see Figure 6. 


\section{Lengthscales in 2D}

The turbulent kinetic energy $\kappa$ and rate of dissipation of turbulent kinetic energy $\varepsilon$ were computed from 1000 time steps equally spaced along $15 \tau$.

The integral lengthscale of turbulence is obtained from the turbulent kinetic energy $\kappa$ and rate of dissipation of turbulent kinetic energy $\varepsilon$ values, as $L=\kappa^{3 / 2} / \varepsilon$. To calculate the turbulent lengthscale $L$ for each particular Re, the space average values of the turbulent energy were computed as $\langle\bar{\kappa}\rangle=\iint_{\Omega} \bar{\kappa} d x d y / \iint_{\Omega} d x d y$ and $\langle\bar{\varepsilon}\rangle=\iint_{\Omega} \bar{\varepsilon} d x d y / \iint_{\Omega} d x d y$, where $\bar{\kappa}=$ $\bar{\kappa}(x, y)$ and $\bar{\varepsilon}=\bar{\varepsilon}(x, y)$ are the time-averaged values at each control volume of the mesh, and $\Omega$ is the $2 \mathrm{D}$ domain. The 1000 samples of the flow dynamic solution used to compute the values of $\bar{\kappa}, \bar{\varepsilon}$, and the lengthscales were divided into 5 subsets of 200 time steps, corresponding to $3 \tau$ each subset. From the five subsets it is computed the standard deviation value for these quantities.

Fig. 10 shows the evolution of the turbulent lengthscale $L$ normalized by the chamber diameter $D$ with the Reynolds numbers with the respective standard deviation. The obtained value for the normalized lengthscale is around 2 for the lower Reynolds numbers, due to the fact that pairs of counter rotating vortices with diameter $D$ are the dominant flow structure in this flow. This lengthscale increases with the Reynolds number, i.e. the larger scales of turbulence are growing with Re. The flow scales are not changed in the studied Re range, as seen from the energy spectrum of turbulence in Fig. 7, although the energy distribution around each flow scale becomes more spread through a range of lengthscales. This spreading of energy is clear in Fig. 7 from the decrease of the energy value of the local peak corresponding to the energy containing scales, $(D / 2)^{-1}$, thus this increase in the $L$ scale is due to a larger interplay between the vortices. 
The turbulence integral time scale $\tau_{t}=\kappa / \varepsilon$ can be transformed into a lengthscale by multiplication with a velocity. Fig. 10 shows the evolution of the lengthscale $\tau_{t} v_{\text {out }}$ with the Reynolds number. It can be seen that the values of $\tau_{t} v_{\text {out }}$ are mainly concentrated between $1.5 D$ and $2 D$. Similar results were already obtained from the analysis of $L$. The values for the turbulence integral time scale, $1.5 D$ to $2 D$, are easily related with the larger vortices, while the lengthscales identified with $L$ between $1.75 D$ and $3 D$ are not so physically sounded.

Turbulence integral lengthscale can also be obtained from two-point cross-correlation of velocity. The cross correlation of dynamic velocity data reveals the scale of the flow structures. The longitudinal integral scale is defined by

$$
L_{11}(x)=\int_{0}^{\infty} \frac{\langle u(x+r, t) u(x, t)\rangle}{\langle u(x, t) u(x, t)\rangle} d r
$$

where $\langle u(x+r, t) u(x, t)\rangle$ is the two-point correlation. The $L_{11}$ is a characteristic lengthscale of the larger eddies ${ }^{2}$. Fig. 11 shows the evolution of the normalized $L_{11}$ along the chamber axis, for several Re. It can be seen that near $x \approx d+D / 2$, the larger eddies identified by $L_{11}$ have a diameter equal to half chamber width. These structures evolve to larger vortices having the same diameter as the chamber width, for $x>2 D$.

\section{Comparison between 2D and 3D CIJs turbulence dynamics}

The analysis of the turbulence scales is also made in a 3D CIJ, which consists of a cylindrical mixing chamber with two opposite injectors placed near the top of the chamber and the outlet in the bottom, as can be seen from Fig. 1b. The CIJ used in this paper has a diameter $D=$ $10 \times 10^{-3} \mathrm{~m}$ and height $H=5 D=50 \times 10^{-3} \mathrm{~m}$. The injectors with diameter $d=1.5 \times$ $10^{-3} \mathrm{~m}$ are placed at $D / 2$ from the top of the mixing chamber.

CFD simulation of 3D CIJs was made for a Reynolds number $\operatorname{Re}=\rho v_{i n j} d / \mu=150$, which is immediately above the transition from steady to chaotic flow regimes that occurs at $\mathrm{Re}=$ 125 in 3D CIJs. This Reynolds number of 150 for 3D geometries is closely related in terms of 
flow regimes with the $2 \mathrm{D}$ case where $\mathrm{Re}=300$. On the comparison of $2 \mathrm{D}$ and $3 \mathrm{D}$ transition Reynolds numbers in opposed jets mixers see Santos et al. ${ }^{28}$.

In Fig. 4, isosurfaces of the $z$ vorticity component are used to show the overall rotation of the flow in the CIJ. The imaging of 3D flows is quite complex, and so only some information on the 3D flow can be obtained from Fig. 4. A more detailed description will be made from previous knowledge on this reactor $29,33,36,37,59-62$. Upon the opposed jets impingement the fluids spread radially forming a pancake like flow structure. In this structure with circular shape and diameter approximately $D$, the two reactants contact on a surface with area $\pi D^{2} / 4$, this represents a fortyfold increase from the initial contact area of the opposed jets: $\pi d^{2} / 4$. The increase of the contact area of the fluids leads to a decrease of the mixing lengthscales of the inverse order, so the initial scale of the jets is reduced by at least one order of magnitude upon the jets impingement ${ }^{63}$. Downstream of the impingement point, vortices engulf this pancake like structure causing oscillations of the flow in the jets impingement point. These vortices form laminas of fluid from the opposed jets that are oriented in the perpendicular direction to the main flow direction towards the outlet as can be seen in Fig. $12^{27}$. The thickness of the laminas is around $3 \times 10^{-6} \mathrm{~m}^{63}$. The vortices formed around the jets, can be seen in Fig. 4, at least the part of the vortices rotating perpendicularly to the $z$-direction below and above the jets. The complete annular vortex around the jet is only seen from other vorticity components that are not shown in Fig. 4.

In the 3D case, one chamber diameter downstream from the injectors the flow has a parabolic profile, whereas in the $2 \mathrm{D}$ case the vortices with diameter $D$ evolve throughout the chamber until the outlet at $4.5 \mathrm{D}$ from the impact point.

Despite the differences between 3D CIJ and the 2D CIJ flows, the flows are quite similar regarding their dynamics. Fig. 12 shows the power spectrum for the $3 \mathrm{D}$ CIJ at $x=d+D / 2$, obtained from $\mathrm{Re}=150 \mathrm{CFD}$ simulation. The wavenumbers were obtained considering the vortices velocity that is calculated from the vortices formation frequency rate and 
lengthscale, $\lambda=f / v_{\text {vortex }}$. The frequency with higher energy for the $3 \mathrm{D}$ CIJ is $\approx 40 \mathrm{~s}^{-1}$, which is associated with the larger vortices seen from PLIF in Fig.12 having diameter approximately $D / 2$. At this location, each vortex is fed from one injector with flow rate $q=$ $\pi(d / 2)^{2} v_{\text {inj }}$, and has volume $V_{\text {vortex }} \approx \pi D_{\text {vortex }}^{2} d_{2} / 6$ where $d_{2}=D / 2^{29}$. From $f=$ $V_{\text {vortex }} / q$ the vortex diameter is $D_{\text {vortex }} \approx 6 \times 10^{-3} \mathrm{~m}$, which is quite close to the size of the vortices seen from PLIF image in Fig. 12. The velocity of these larger vortices can be estimated from both diameter and frequency: $v_{\text {vortex }}=D_{\text {vortex }} f \approx 0.24 \mathrm{~m} / \mathrm{s}$. The frequency of the injection scale, that occurs at $f \approx 120 \mathrm{~s}^{-1}$, can be related to a lengthscale through $\delta=v_{\text {vortex }} / f \approx 1.9 \times 10^{-3} \mathrm{~m}$, which yields a value close to the injectors diameter $d=$ $1.5 \times 10^{-3} \mathrm{~m}$

The wavenumber in Fig. 12 is obtained from the frequency normalized by the average vortices velocity, $v_{\text {vortex }}=0.24 \mathrm{~m} / \mathrm{s}$. From the plot in Fig. 12 it is seen the same fundamental mechanism of energy transfer between scales that was seen for $2 \mathrm{D}$ turbulence: there is an injection scale of energy that is smaller than the energy containing scales, and so energy transfer is inversed in the inertial subrange from the smaller scale, $d$, to the larger scale, $D / 2$. Nevertheless, the mixing scales, which start from $\delta=d$, are always reduced, even when the turbulence scales grow, as can be seen in Fig. 12. And so, although the turbulence scale grows to $D / 2=5 \times 10^{-3} \mathrm{~m}$ the mixing scales are reduced from $\delta_{0}=d=$ $1.5 \times 10^{-3} \mathrm{~m}$ by a factor of 40 in the impingement region to $\approx \delta_{0} / 40=37.5 \times 10^{-6} \mathrm{~m}^{63}$. After the flow develops into a parabolic profile where the shear rate is $\dot{\gamma} \approx 2 v_{\text {out }} /(D / 2)$ over a mixing chamber distance of $4 D$, and thus $\dot{\gamma} t \approx \dot{\gamma}\left(4 D / v_{\text {out }}\right)=16$ and so the mixing scale further reduces to $\delta=\delta_{0} /(40 \dot{\gamma} t) \approx 37.5 \times 10^{6} / 16 \approx 2 \times 10^{-6} \mathrm{~m}$.

The main difference of the 3D CIJ flow and 2D CIJ flow is on the jets contacting. In the 3D CIJs the opposed jets impinge each other and after the fluid spreads radially from the impingement point, while in the $2 \mathrm{D}$ CIJs the jets bend towards the outlet before contacting and so there is no deceleration of the jets upon impinging each other ${ }^{28}$. The radial spreading 
of the jets in the 3D CIJs causes the flow dynamics to dissipate at a distance $D$ from the impingement point, after which point the flow is fully developed with a parabolic velocity profile. In the $2 \mathrm{D}$ CIJs the fact that the flow is constrained in one dimension hinders dissipation mechanisms promoting a dynamic evolution of vortices throughout the mixing chamber at least up to the outlet of simulated domain, $4.5 \mathrm{D}$. So, the 3D CIJs have a faster reduction of scales when the jets impinge, but the flow dynamics dissipates in a shorter region of the mixing chamber. Despite these differences in the flow spatial evolution the dynamics of 2D and 3D CIJs have the common aspect of being caused by the evolution of a shorter scale jet, $d$, that feeds larger vortices, $D$ or $D / 2$. Due to this fact both 2D and 3D flow dynamics are well described within the framework of $2 \mathrm{D}$ turbulence as seen from the power spectra of both Fig. 7 and Fig. 12 where the power spectra from both geometries is plotted. The larger concentration of turbulent kinetic energy in the 2D CIJs is also clear from the power spectra comparison in Fig. 12. Similar flow regime to the one simulated with the 2D model have been experimentally observed with PLIF in prismatic mixing chambers if the depth was at least $D / 2^{48,64}$.

\section{CONCLUSIONS}

2D turbulence was studied from the 2D CFD simulation of an opposed jets mixer. This mixer is a simplification of actual industrial reactors used for example in crystallization and polymer processing $52,65-68$. The following observations are made from the energy distribution of turbulence for 2D flow:

- the energy is injected at a small scale, which is the smallest observable coherent structure from the flow maps, the injectors' width;

- the energy is transferred from the injection scale to a larger hydrodynamic scale through the inertial subrange, where the size of the larger scales is the diameter of vortices that occupy the entire section of the mixing chamber; 
- for scales below the injection scale turbulence energy is dissipated by viscosity;

- in the flow region dominated by the jets contacting there is an equilibrium of energy transfer from the jet scale to the vortices scale.

From the energy budget of kinetic and turbulent energy dissipation the integral scales of turbulence were computed, and had approximately the dimension of a pair of counter-rotating vortices, $2 D$. The scales obtained from the power spectrum of velocity at a point and from the cross correlation between points at different locations, in this case the longitudinal integral scale, yielded approximately $D$.

The same methodology of flow analysis is applied to an actual 3D geometry, equal to mixers with industrial applications. In these mixers the flow is extremely conditioned by the fact that energy is injected in a narrow section of the mixing chamber defined by the opposite injectors, and so the flow is mainly defined in the plane containing the mixing chamber axis and the axes of the injectors. The flow at the 3D CIJ was at a transition flow regime and has some of the same features than the 2D flow, namely the injection of energy at a smaller scale and its transport through the inertial subrange towards the energy containing scales. Thus, the flow dynamics at 3D CIJs under transitional flow regimes is well-described within the framework of $2 \mathrm{D}$ turbulence as described by Kraichnan ${ }^{1,3}$. This is to our knowledge the first demonstration of 2D turbulence in an industrial mixer/reactor. These mixers have been used in industry, namely for reactive polymerization in RIM processes, where the viscosity range of operation makes it impossible to design equipment for turbulent mixing under the classic scenario of scale reduction by vortex stretching. The $2 \mathrm{D}$ turbulence mechanism of injecting energy at a smaller scale that evolves to larger flow scale, was shown to be associated in these flows with a fast reduction of mixing scales that is a necessary condition for chemical reaction to take place. The 2D turbulence description is a useful tool for the assessment of the design 
of CIJs because the energy spectra is linked to all the flow scales involved in the mixing process.

\section{AKNOWLEDGMENTS}

This work was financed by Project “AIProcMat@N2020 - Advanced Industrial Processes and Materials for a Sustainable Northern Region of Portugal 2020", with the reference NORTE01-0145-FEDER-000006, supported by Norte Portugal Regional Operational Programme (NORTE 2020), under the Portugal 2020 Partnership Agreement, through the European Regional Development Fund (ERDF) and Projects POCI-01-0145-FEDER-006984 Associate Laboratory LSRE-LCM and PTDC/QEQ-FTT/0041/2014 funded by ERDF through COMPETE2020 - Programa Operacional Competitividade e Internacionalização (POCI) and by national funds through FCT - Fundação para a Ciência e a Tecnologia.

\section{NOTATION}

Roman letters

$A_{\text {vortex }}$ - vortex area $\left[\mathrm{m}^{2}\right]$

C - Courant number $[-]$,

$d$ - injectors width, for 2D CIJ, or diameter for 3D CIJ [m],

$D$ - mixing chamber width, for 2D CIJ, or diameter for 3D CIJ [m],

$D_{\text {vortex }}$ - vortex diameter $[\mathrm{m}]$,

$E$ - power energy spectrum $\left[\mathrm{m}^{2}\right]$,

$f-$ frequency $\left[\mathrm{s}^{-1}\right]$,

$f_{\text {form }}$ - vortices formation frequency $\left[\mathrm{s}^{-1}\right]$,

$H$ - mixing chamber length $[\mathrm{m}]$,

$l_{K}$ - Kolmogorov lengthscale $[\mathrm{m}]$, 
$L$ - integral lengthscale of turbulence $[\mathrm{m}]$,

$L_{11}$ - longitudinal integral scale $[\mathrm{m}]$,

$n$ - Hamming window function width [-],

$p$-pressure $[\mathrm{Pa}]$,

$q$ - flow rate $\left[\mathrm{m}^{3} \mathrm{~s}^{-1}\right]$,

$r$ - radius (radial coordinate) $[\mathrm{m}]$,

Re - Reynolds number [-],

$s_{i j}$ - fluctuating rate of strain tensor $\left[\mathrm{s}^{-1}\right]$,

$t$ - time $[s]$,

$\boldsymbol{u}$ - velocity vector $[\mathrm{m} / \mathrm{s}]$,

$u$ - x-component of the velocity $[\mathrm{m} / \mathrm{s}]$,

$\bar{u}$ - x-component of the average velocity $[\mathrm{m} / \mathrm{s}]$,

$u^{\prime}$ - x-component of the fluctuating velocity $[\mathrm{m} / \mathrm{s}]$,

$v$ - kinematic viscosity $\left[\mathrm{m}^{2} / \mathrm{s}\right]$,

$V_{\text {vortex }}$ - vortex volume $\left[\mathrm{m}^{3}\right]$

$w$ - z-component of the velocity $[\mathrm{m} / \mathrm{s}]$,

$W$ - Hamming window function $[-]$,

$x$ - x-coordinate $[\mathrm{m}]$,

$y$ - y-coordinate $[\mathrm{m}]$,

$z$ - z-coordinate $[\mathrm{m}]$,

Greek letters

$\alpha$ - Hamming window function parameter $[-]$,

$\beta$ - Hamming window function parameter [-],

$\dot{\gamma}$ - shear rate $\left[\mathrm{s}^{-1}\right]$,

$\delta$ - mixing scale $[\mathrm{m}]$, 
$\delta_{0}$ - mixing scale next to the injectors $[\mathrm{m}]$,

$\delta_{t}$ - time step $[\mathrm{s}]$

$\delta_{x}$ - mesh element size (edge length) [m],

$\varepsilon$ - turbulent kinetic energy dissipation rate $\left[\mathrm{m}^{2} \mathrm{~s}^{-3}\right]$

$\bar{\varepsilon}$ - time average turbulent kinetic energy dissipation rate $\left[\mathrm{m}^{2} \mathrm{~s}^{-3}\right]$,

$\langle\vec{\varepsilon}\rangle$ - space and time average turbulent kinetic energy dissipation rate $\left[\mathrm{m}^{2} \mathrm{~s}^{-3}\right]$,

$\lambda$ - wavenumber $\left[\mathrm{m}^{-1}\right]$,

$\lambda_{\text {inj }}^{-1}$ - energy injection scale $[\mathrm{m}]$,

$\left\langle\kappa_{i n j}\right\rangle$ - space average kinetic energy injected to the system $\left[\mathrm{m}^{2} \mathrm{~s}^{-2}\right]$,

$\bar{\kappa}$ - time average turbulent kinetic energy $\left[\mathrm{m}^{2} \mathrm{~s}^{-2}\right]$,

$\langle\bar{\kappa}\rangle$ - space and time average turbulent kinetic energy $\left[\mathrm{m}^{2} \mathrm{~s}^{-2}\right]$,

$\kappa^{*}$ - normalized turbulent kinetic energy $[-]$,

$\overline{\kappa^{*}}$ - normalized time average turbulent kinetic energy [-],

$\mu$ - dynamic viscosity $[\mathrm{Pa} \cdot \mathrm{s}]$,

$\rho$ - fluid density $\left[\mathrm{kg} / \mathrm{m}^{3}\right]$,

$\tau$ - passage time $[\mathrm{s}]$,

$\tau_{t}$ - turbulence integral time $[\mathrm{s}]$,

$v$ - y-component of the velocity $[\mathrm{m} / \mathrm{s}]$,

$v^{\prime}$ - y-component of the fluctuating velocity $[\mathrm{m} / \mathrm{s}]$,

$\bar{v}$ - y-component of the average velocity $[\mathrm{m} / \mathrm{s}]$,

$v_{i n j}$ - injectors (inlet) average velocity $[\mathrm{m} / \mathrm{s}]$,

$v_{\max }$ - maximum velocity (at the injectors centre) $[\mathrm{m} / \mathrm{s}]$,

$v_{\text {out }}$ - outlet average velocity $[\mathrm{m} / \mathrm{s}]$,

$v_{\text {vortex }}$ - vortex velocity $[\mathrm{m} / \mathrm{s}]$,

$\Omega$-domain. 


\section{LITERATURE CITED}

1. Boffetta G, Ecke RE. Two-Dimensional Turbulence. Annu Rev Fluid Mech. 2012;44(1):427-51.

2. Pope SB. Turbulent flows: Cambridge University Press; 2000.

3. Kraichnan RH. Inertial Ranges in Two-Dimensional Turbulence. Phys Fluids. $1967 ; 10(7): 1417$.

4. Hidema R, Suzuki H, Hisamatsu S, Komoda Y. Characteristic scales of twodimensional turbulence in polymer solutions. AIChE J. 2014;60(5):1854-62.

5. Batchelor GK. Computation of the Energy Spectrum in Homogeneous TwoDimensional Turbulence. Phys Fluids. 1969;12(12):II-233.

6. Amarouchene Y, Kellay H. Polymers in 2D turbulence: suppression of large scale fluctuations. Phys Rev Lett. 2002;89(10):104502.

7. Couder Y, Chomaz JM, Rabaud M. On the Hydrodynamics of Soap Films. Physica D. 1989;37(1-3):384-405.

8. Gharib M, Derango P. A liquid film (soap film) tunnel to study two-dimensional laminar and turbulent shear flows. Physica D. 1989;37(1):406-16.

9. Kellay H, Wu XL, Goldburg WI. Experiments with Turbulent Soap Films. Phys Rev Lett. 1995;74(20):3975-8.

10. Vorobieff P, Rivera M, Ecke RE. Soap film flows: Statistics of two-dimensional turbulence. Phys Fluids. 1999;11(8):2167-77.

11. Kellay H, Wu XL, Goldburg WI. Vorticity measurements in turbulent soap films. Phys Rev Lett. 1998;80(2):277-80.

12. Kellay H, Goldburg WI. Two-dimensional turbulence: a review of some recent experiments. Rep Prog Phys. 2002;65(5):845-94. 
13. Rivera M, Vorobieff $\mathrm{P}$, Ecke RE. Turbulence in flowing soap films: Velocity, vorticity, and thickness fields. Phys Rev Lett. 1998;81(7):1417-20.

14. Rivera MK, Daniel WB, Chen SY, Ecke RE. Energy and enstrophy transfer in decaying two-dimensional turbulence. Phys Rev Lett. 2003;90(10):104502.

15. Sommeria J. Experimental-Study of the Two-Dimensional Inverse Energy Cascade in a Square Box. J Fluid Mech. 1986;170:139-68.

16. Paret J, Marteau D, Paireau O, Tabeling P. Are flows electromagnetically forced in thin stratified layers two dimensional? Phys Fluids. 1997;9(10):3102.

17. Rivera MK, Ecke RE. Pair dispersion and doubling time statistics in two-dimensional turbulence. Phys Rev Lett. 2005;95(19):194503.

18. Cardoso O, Marteau D, Tabeling P. Quantitative experimental study of the free decay of quasi-two dimensional turbulence. Phys Rev E. 1994;49(1):454-61.

19. Bondarenko N, Gak E, Gak M. Application of MHD effects in electrolytes for modeling vortex processes in natural phenomena and in solving engineering-physical problems. J Eng Phys Thermophys. 2002;75:1234-47.

20. Marteau D, Cardoso O, Tabeling P. Equilibrium states of two-dimensional turbulence: an experimental study. Phys Rev E. 1995;51:5124-7.

21. Dolzhanskii FV, Krymov VA, Manin DY. An advanced experimental investigation of quasi-two dimensional shear flow. J Fluid Mech. 1992; 241:705-22.

22. Akkermans RAD, Kamp LPJ, Clercx HJH, Heijst GJFv. Intrinsic three-dimensionality in electromagnetically driven shallow flows. Europhys Lett. 2008;83(2):24001.

23. Shats M, Byrne D, Xia H. Turbulence decay rate as a measure of flow dimensionality. Phys Rev Lett. 2010;105(26):264501.

24. Chomaz JM. The dynamics of a viscous soap film with soluble surfactant. J Fluid Mech. 2001;442:387-409. 
25. Rivera M, Wu XL. External dissipation in driven two-dimensional turbulence. Phys Rev Lett. 2000;85(5):976-9.

26. Rivera $\mathrm{M}, \mathrm{Wu} \mathrm{XL}$. Homogeneity and the inertial range in driven two-dimensional turbulence. Phys Fluids. 2002;14(9):3098-108.

27. Fonte CP, Sultan MA, Santos RJ, Dias MM, Lopes JCB. Flow imbalance and Reynolds number impact on mixing in Confined Impinging Jets. Chem Eng J. 2015;260:31630.

28. Santos RJ, Teixeira AM, Erkoç E, Sultan MA, Karpinska AM, Dias MM, et al. Validation of a 2D CFD Model for Hydrodynamics' Studies in CIJ Mixers. Int J Chem React Eng. 2010;8.

29. Santos RJ, Erkoç E, Dias MM, Lopes JCB. Dynamic Behavior of the Flow Field in a RIM Machine Mixing Chamber. AIChE J. 2009;55(6):1338-51.

30. Malguarnera SC, Suh NP. Liquid injection molding I. An investigation of impingement mixing. Polym Eng Sci. 1977;17(2):111-5.

31. Lee LJ, Ottino JM, Ranz WE, Macosko CW. Impingement Mixing in Reaction Injection-Molding. Polym Eng Sci. 1980;20(13):868-74.

32. Tucker CL, Suh NP. Mixing for reaction injection molding. I. Impingement mixing of liquids. Polym Eng Sci. 1980;20(13):875-86.

33. Wood P, Hrymak A, Yeo R, Johnson D, Tyagi A. Experimental and Computational Studies of the Fluid-Mechanics in an Opposed Jet Mixing Head. Phys Fluids A: Fluid. 1991;3(5):1362-8.

34. Johnson DA. Experimental and numerical examination of confined laminar opposed jets part I. Momentum imbalance. Int Commun Heat Mass. 2000;27(4):443-54.

35. Johnson DA. Experimental and numerical examination of confined laminar opposed jets part II. Momentum balancing. Int Commun Heat Mass. 2000;27(4):455-63. 
36. Johnson DA, Wood PE. Self-sustained oscillations in opposed impinging jets in an enclosure. Can J Chem Eng. 2000;78(5):867-75.

37. Johnson DA, Wood PE, Hrymak AN. The effect of geometrical parameters on the flow field of an opposed jet RIM mix head: Equal flow and matched fluids. Can J Chem Eng. 1996;74(1):40-8.

38. Trautmann P, Piesche M. Experimental Investigations on the Mixing Behaviour of Impingement Mixers for Polyurethane Production. Chem Eng Technol. 2001;24(11):1193-7.

39. Santos RJ, Teixeira AM, Costa MRPFN, Lopes JCB. Operational and design study of RIM machines. Int Polym Proc. 2002;17(4):387-94.

40. Santos RJ, Erkoç E, Dias MM, Teixeira AM, Lopes JCB. Hydrodynamics of the mixing chamber in RIM: PIV flow-field characterization. AIChE J. 2008;54(5):1153-63.

41. Santos RJ, Teixeira AM, Lopes JCB. Study of mixing and chemical reaction in RIM. Chem Eng Sci. 2005;60(8-9):2381-98.

42. Teixeira AM, Santos RJ, Rui M, Costa PFN, Lopes JCB. Hydrodynamics of the mixing head in RIM: LDA flow-field characterization. AIChE J. 2005;51(6):1608-19.

43. Fonte CP, Sultan MA, Santos RJ, Dias MM, Lopes JCB. An Elastic Analog Model for Controlling the Impingement Point Position in Confined Impinging Jets. AIChE J. 2016;62(6):2200-12.

44. Gobby D, Angeli P, Gavriilidis A. Mixing Characteristics of T-type Microfluidic Mixers. J Micromech Microeng. 2001;11(2):126-32.

45. Engler M, Kockmann N, Kiefer T, Woias P. Numerical and experimental investigations on liquid mixing in static micromixers. Chem Eng J. 2004;101(1-3):315-22.

46. Bothe D, Stemich C, Warnecke HJ. Fluid mixing in a T-shaped micro-mixer. Chem Eng Sci. 2006;61(9):2950-8.

47. Bothe D, Stemich C, Warnecke HJ. Computation of scales and quality of mixing in a T-shaped microreactor. Comput Chem Eng. 2008;32(1-2):108-14. 
48. Sultan MA, Fonte CP, Dias MM, Lopes JCB, Santos RJ. Experimental study of flow regime and mixing in T-jets mixers. Chem Eng Sci. 2012;73:388-99.

49. Nguyen NT, Wu ZG. Micromixers - a review. J Micromech Microeng. 2005;15(2):R1R16.

50. Fonte CP, Santos RJ, Dias MM, Lopes JCB. Quantification of Mixing in RIM Using a Non-Diffusive Two-Phase Flow Numerical Model. Int J Chem React Eng. 2011;9(1):A114.

51. Erkoç E, Santos RJ, Nunes MI, Dias MM, Lopes JCB. Mixing dynamics control in RIM machines. Chem Eng Sci. 2007;62(18-20):5276-81.

52. Gomes NMO, Fonte CP, Sousa CC, Mateus AJ, Bártolo PJ, Dias MM, et al. Real time control of mixing in Reaction Injection Moulding. Chem Eng Res Des. 2016;105:31-43.

53. Tu GY, Li WF, Du KJ, Huang GF, Wang FC. Onset and influencing factors of deflecting oscillation in planar opposed jets. Chem Eng J. 2014;247:125-33.

54. Shi Z-h, Li w-f, Du K-j, Liu H-f, Wang F-c. Experimental study of mixing enhancement of viscous liquids in confined impinging jets reactor at low jet Reynolds numbers. Chem Eng Sci. 2015;138:216-26.

55. Li WF, Qian WW, Yu GS, Liu HF, Wang FC. Experimental Study of Oscillation Behaviors in Confined Impinging Jets Reactor Under Excitation. AIChE J. 2015;61(1):33341.

56. Li WF, Huang GF, Tu GY, Liu HF, Wang FC. Experimental Study of Oscillation of Axisymmetric Turbulent Opposed Jets with Modulated Airflow. AIChE J. 2013;59(12):482838.

57. Tu GY, Li WF, Du KJ, Wang FC. Experimental investigation of deflecting oscillation in T-jets reactor. Chem Eng Sci. 2014;116:734-44.

58. Tu GY, Li WF, Qian WW, Shi ZH, Liu HF, Wang FC. Experimental study on oscillation behaviors in T-jets reactor with excitation. Chem Eng Sci. 2015;134:67-75. 
59. Nakamura S, Brodkey RS, editors. Direct and Large Eddy Simulation of the ThreeDimensional Unsteady Flows in the Counter-Jet Mixing Vessel. ASME Fluids Engineering Summer Conference; 2000; Boston, Massachusetts.

60. Unger DR, Muzzio FJ, Brodkey RS. Experimental and Numerical Characterization of Viscous Flow and Mixing in an Impinging Jet Contactor. The Canadian Journal of Chemical Engineering. 1998;76(3):546-55.

61. Zhao Y, Brodkey RS. Averaged and time-resolved, full-field (three-dimensional), measurements of unsteady opposed jets. Can J Chem Eng. 1998;76(3):536-45.

62. Sandell D, Macosko CW, Ranz WE. Visualization technique for studying impingement mixing at representative Reynolds numbers. Polymer Process Engineering. 1985;3:57-70.

63. Santos RJ, Sultan MA. State of the Art of Mini/Micro Jet Reactors. Chem Eng Technol. 2013;36(6):937-49.

64. Sultan MA, Krupa K, Fonte CP, Nunes MI, Dias MM, Lopes JCB, et al. HighThroughput T-Jets Mixers: An Innovative Scale-Up Concept. Chem Eng Technol. 2013;36(2):323-31.

65. Johnson BK, Prud'homme RK. Mechanism for rapid self-assembly of block copolymer nanoparticles. Phys Rev Lett. 2003;91(11):118302.

66. Lince F, Marchisio DL, Barresi AA. Smart mixers and reactors for the production of pharmaceutical nanoparticles: Proof of concept. Chem Eng Res Des. 2009;87(4):543-9.

67. Kügler RT, Kind M. Experimental study about plugging in confined impinging jet mixers during the precipitation of strontium sulfate. Chemical Engineering and Processing: Process Intensification. 2016;101:25-32.

68. Metzger L, Kind M. Influence of mixing on particle formation of fast precipitation reactions-A new coarse graining method using CFD calculations as a "measuring" instrument. Chemical Engineering Research and Design. 2016. 
(a)

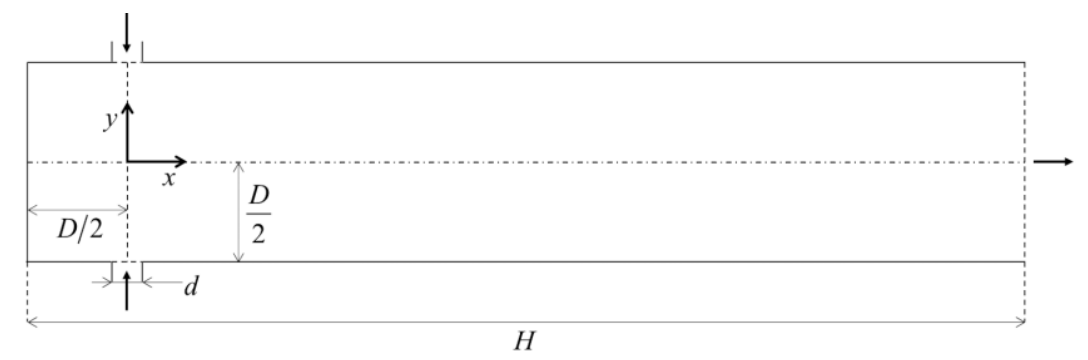

(b)

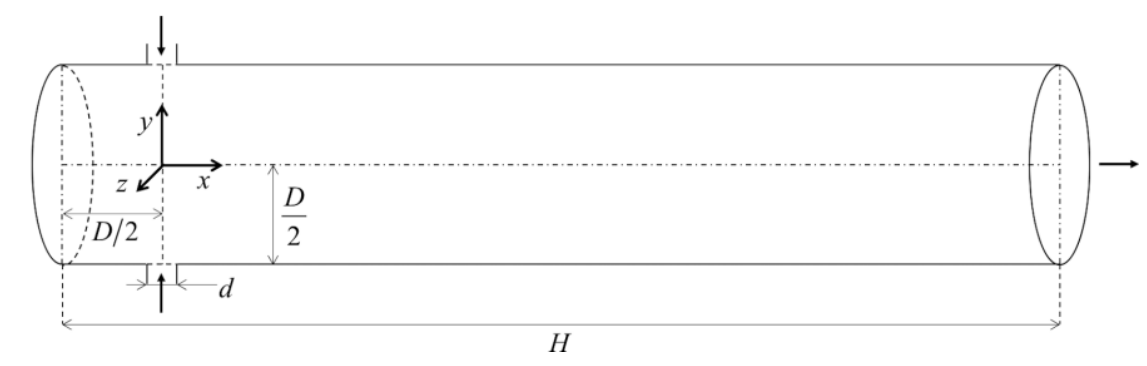

Figure 1. Geometries and dimensions of: (a) CIJ 2D reactor and (b) CIJ 3D reactor. 
(a)

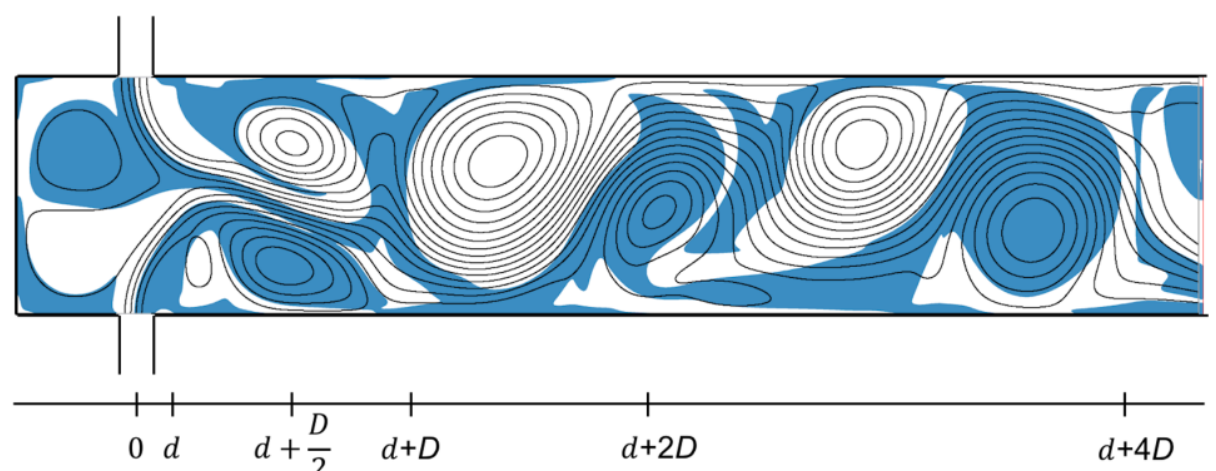

(b)

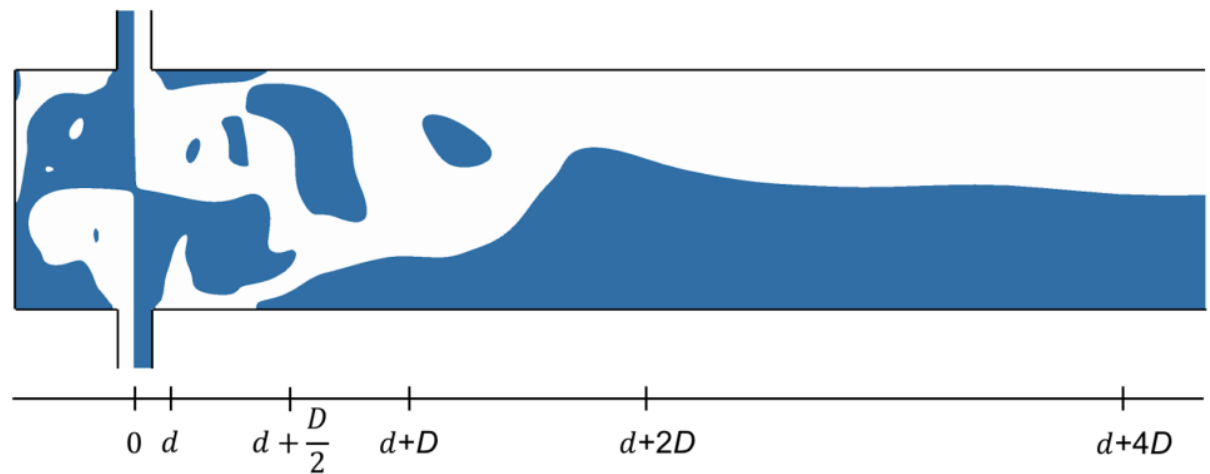

Figure 2. Streamlines and vorticity sign (blue for negative values) (a) CIJ 2D reactor and (b) CIJ 3D reactor. 


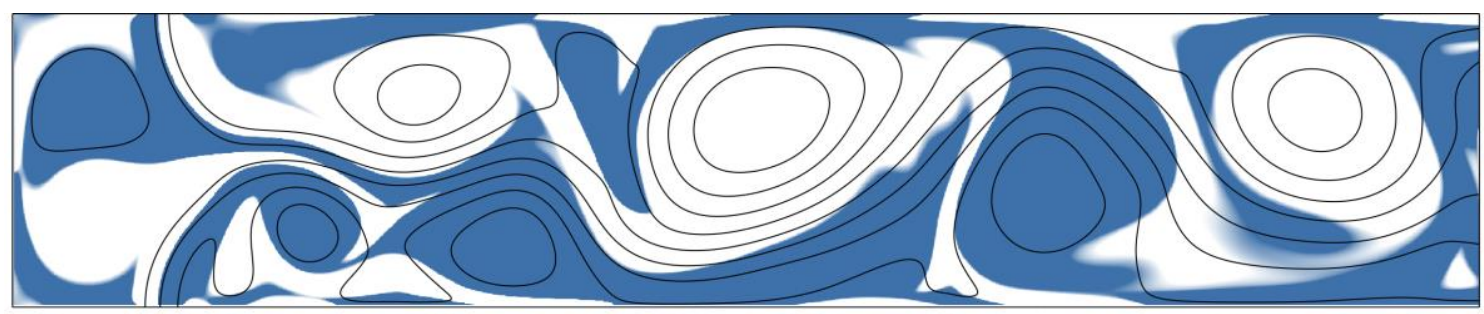

(a)

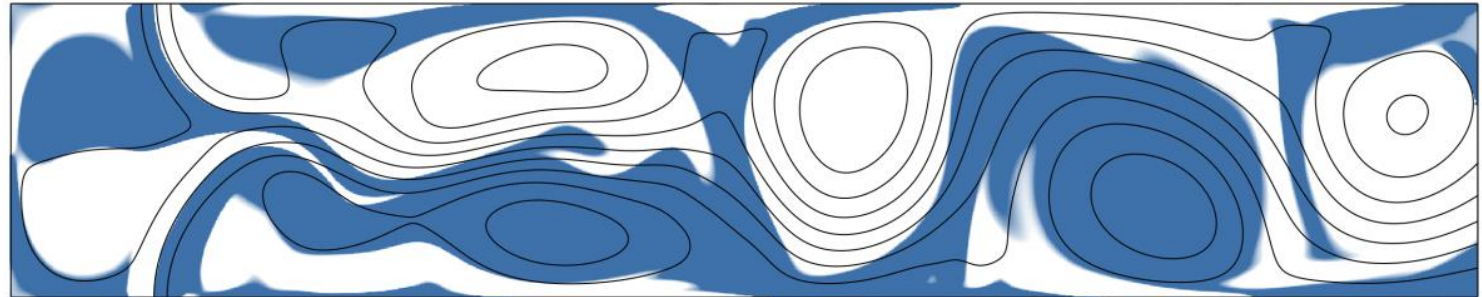

(b)

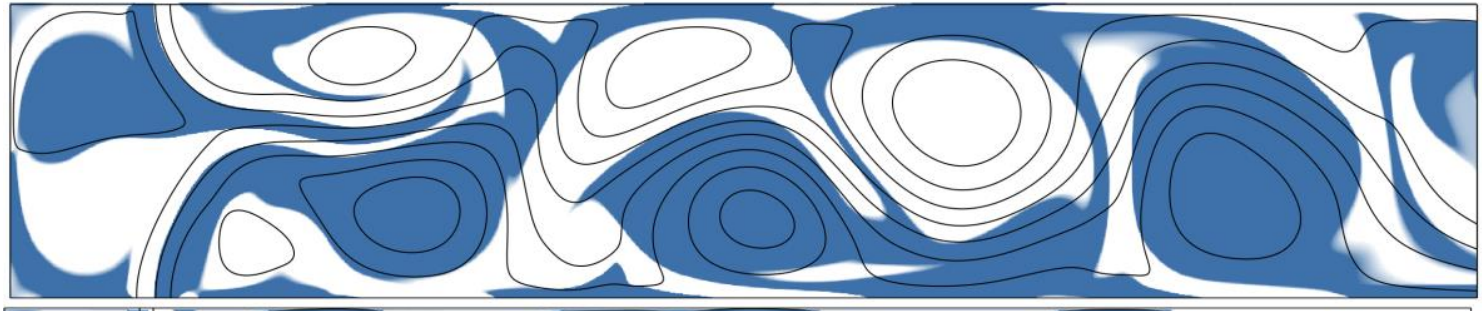

(c)

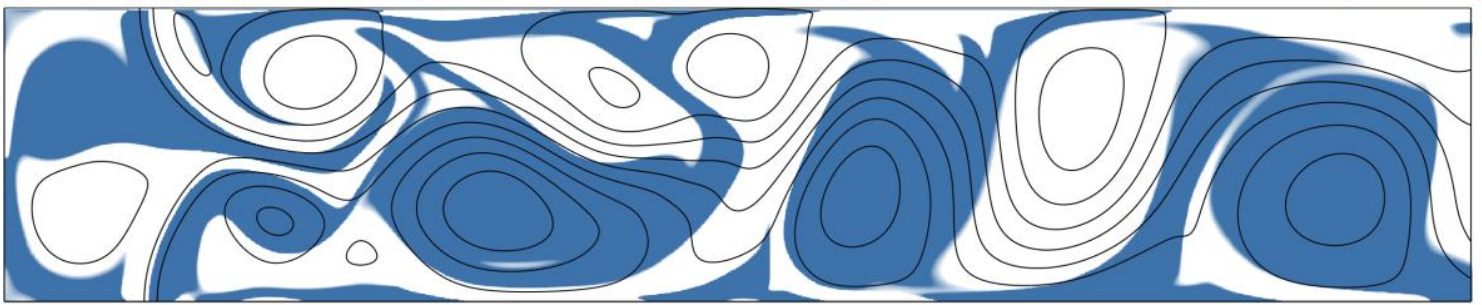

(d)

Figure 3. Streamlines and vorticity sign (blue for negative values) in a CIJ 2D reactor simulation over a period of $0.24 \mathrm{~T}$. 


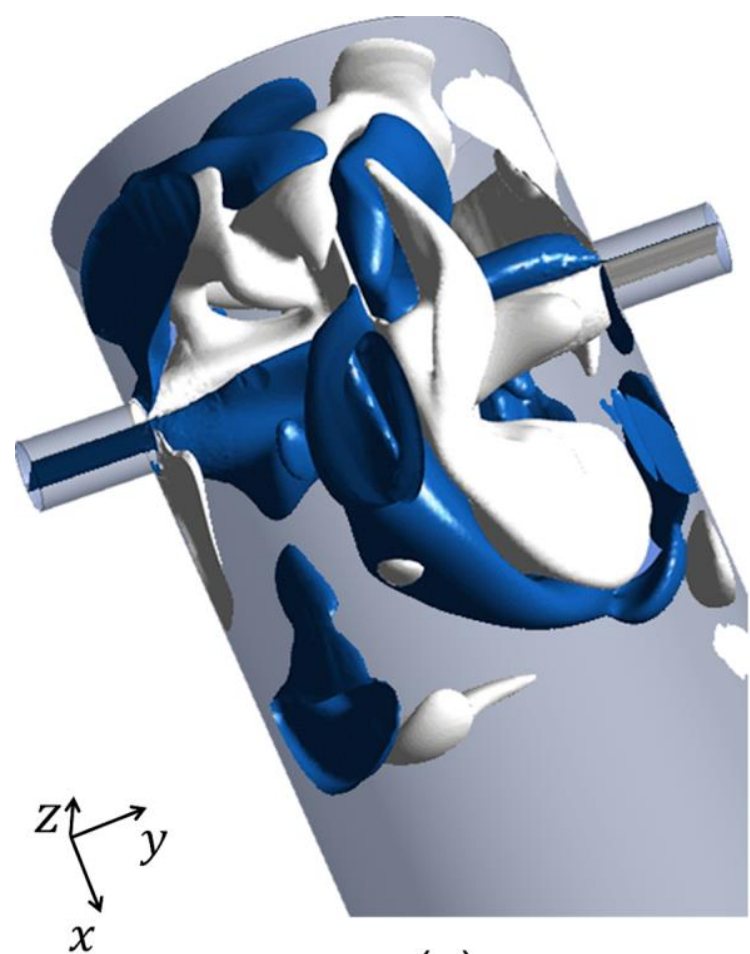

(a)

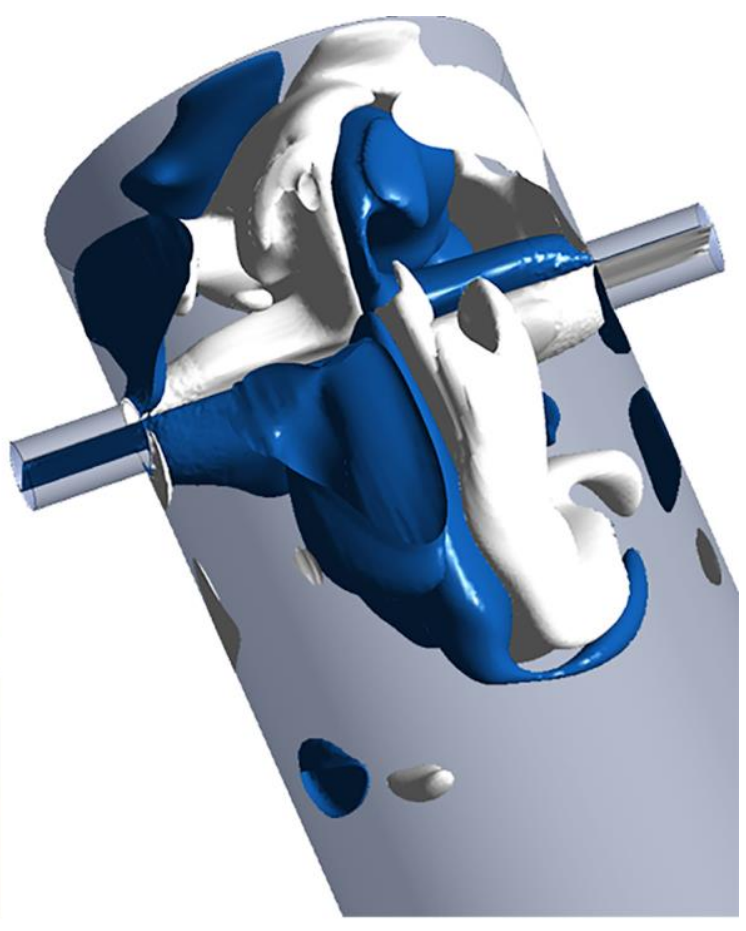

(b)

Figure 4. Streamlines (a and c) and third component of vorticity isosurfaces (c and d) in a CIJ 3D reactor for a numerical simulation in two different moments separated by $0.02 \mathrm{~T}$. 


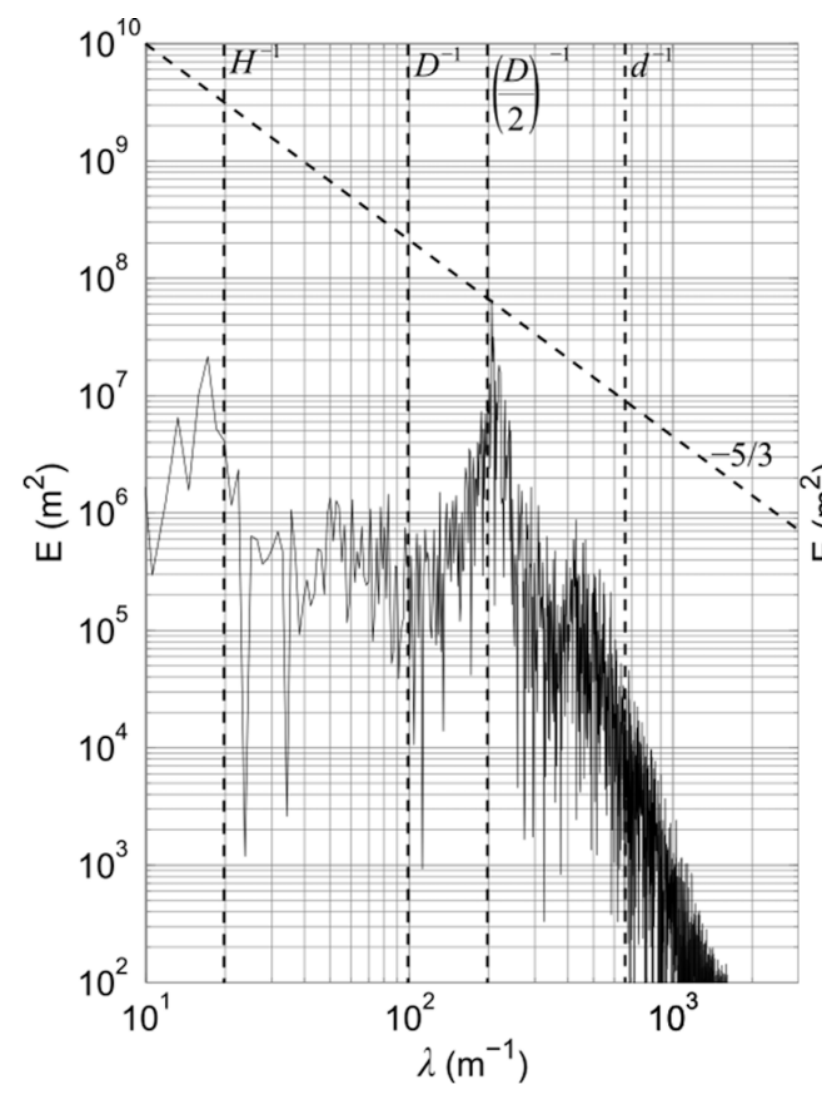

(a)

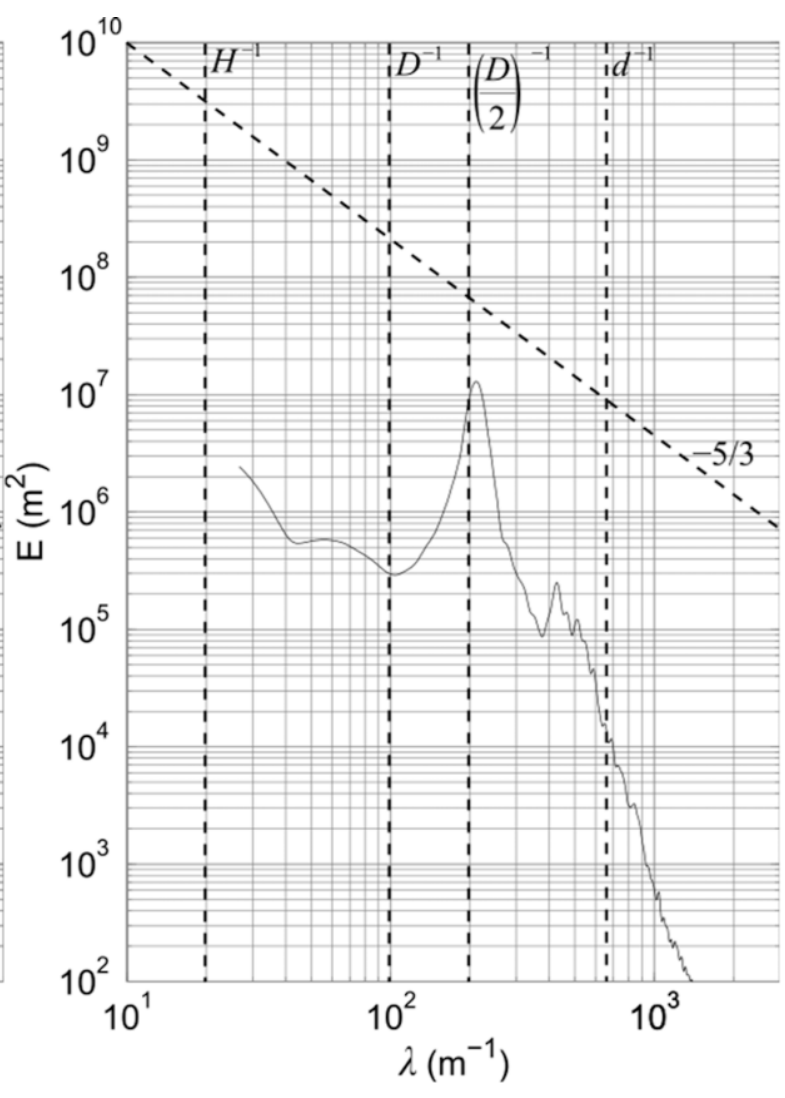

(b)

Figure 5. Power spectrum at impact point, i.e., $(x, y) 5(0,0)$ in CIJ 2D reactor: (a) original and (b) filtered with the

Hamming function. 


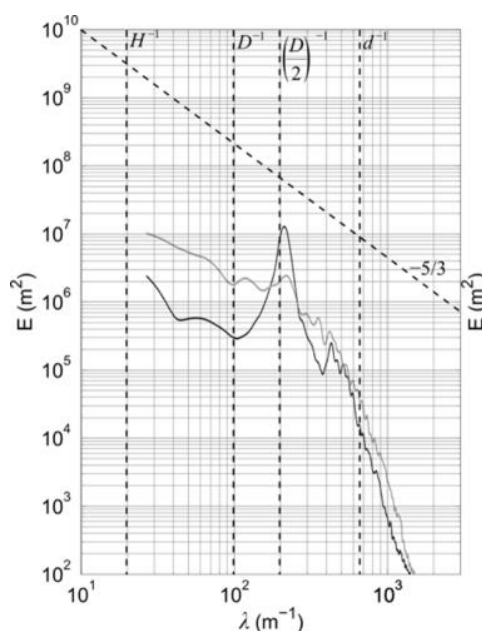

(a) $x=0$

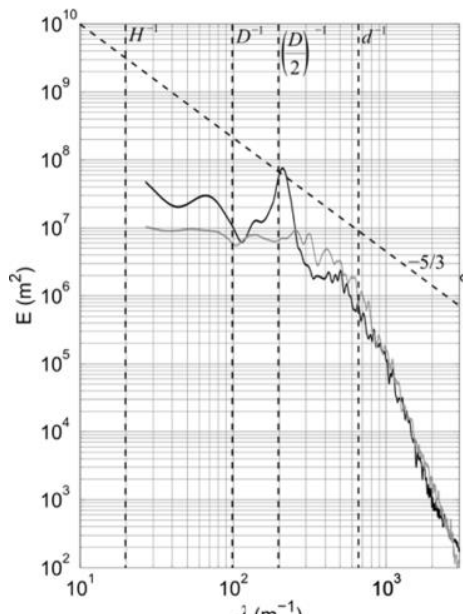

(d) $x=d+2^{-3} D$

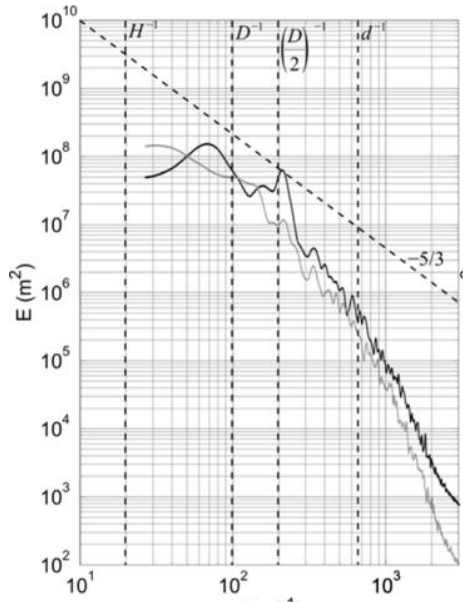

(g) $x=d+D$

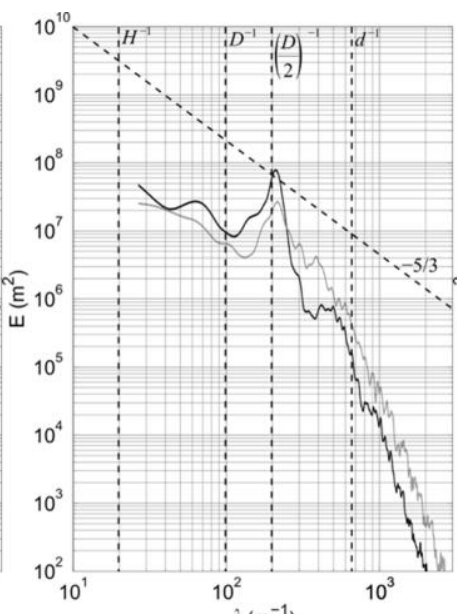

(b) $x=d$

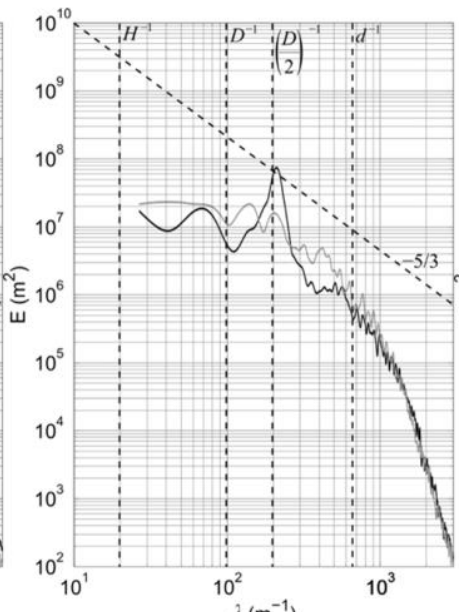

(e) $x=d+2^{-2} D$

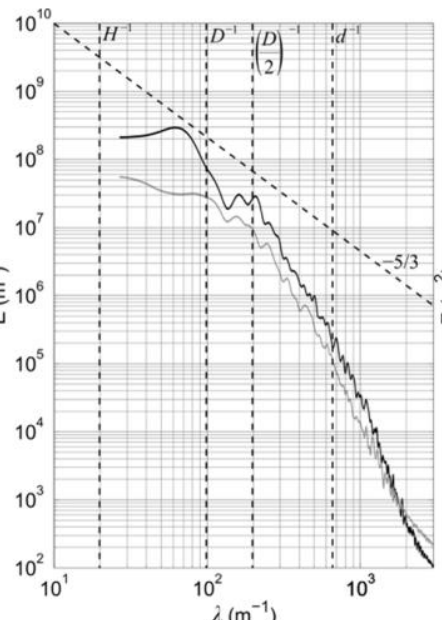

(h) $x=d+2 D$

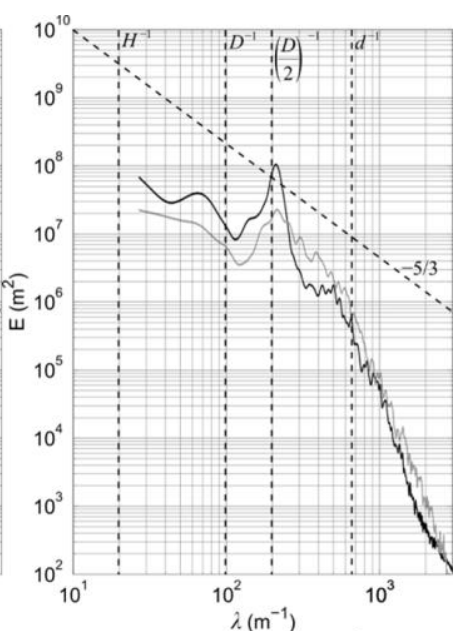

(c) $x=d+2^{-4} D$

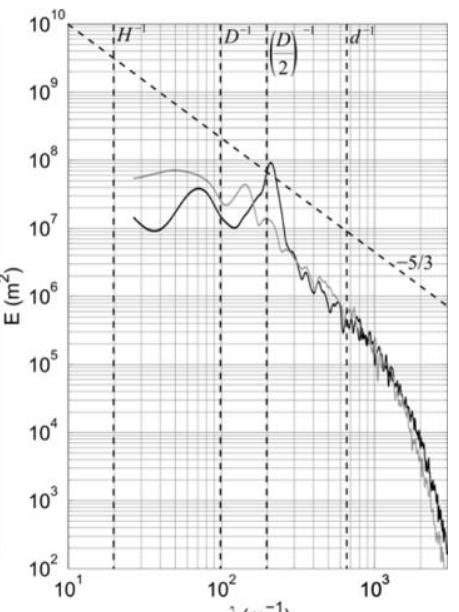

(f) $x=d+2^{-1} D$

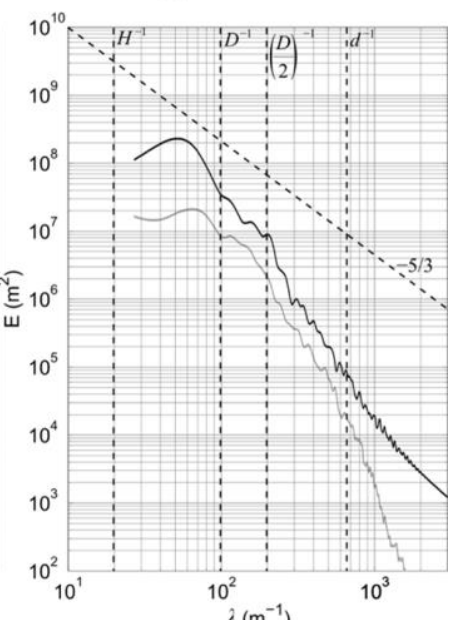

(i) $x=d+2^{2} D$

Figure 6. Power spectra of velocity components (grey for u0 and black for v0) at several locations on y50 axis of

CIJ 2D reactor, with $\operatorname{Re} 5300$. 


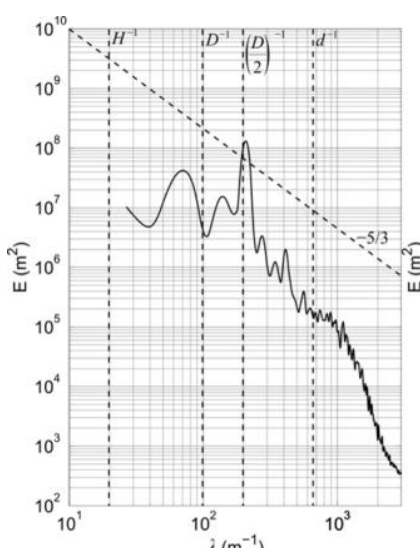

(a) $\stackrel{\lambda\left(\mathrm{m}^{-1}\right)}{\mathrm{Re}}=200$

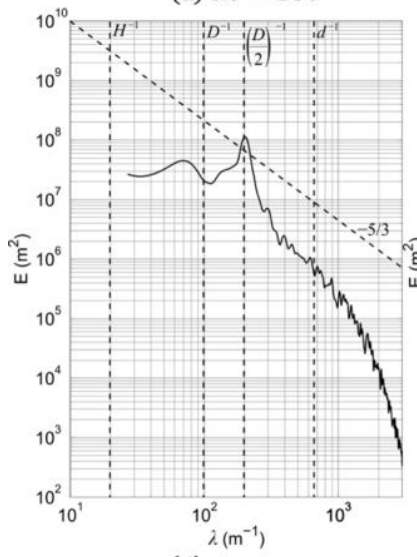

(d) $\operatorname{Re}=400$

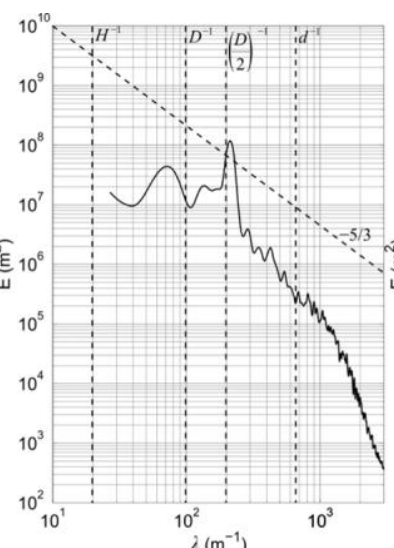

(b) $\operatorname{Re}=250$

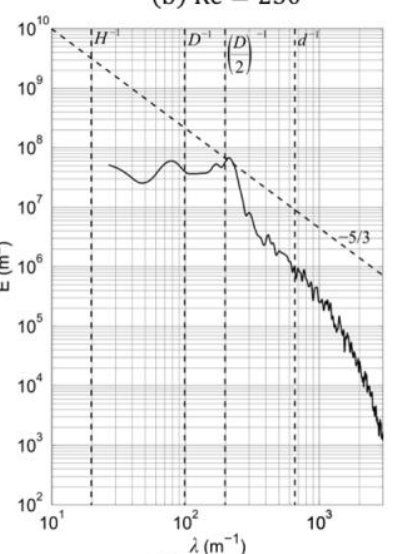

(e) $\operatorname{Re}=500$

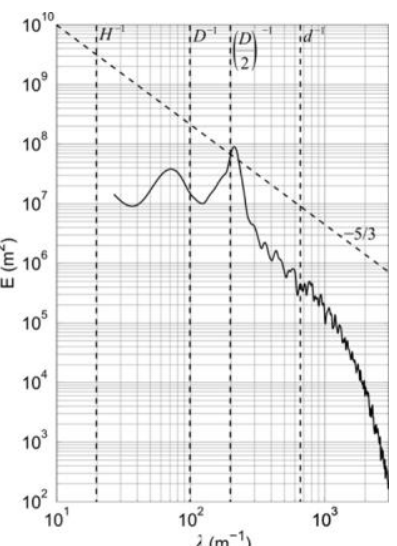

(c) $\operatorname{Re}=300$

Figure 7. Power spectra at $(0, \mathrm{~d} 1 \mathrm{D} / 2)$ from the $2 \mathrm{D}$ CFD simulations for several Re. 


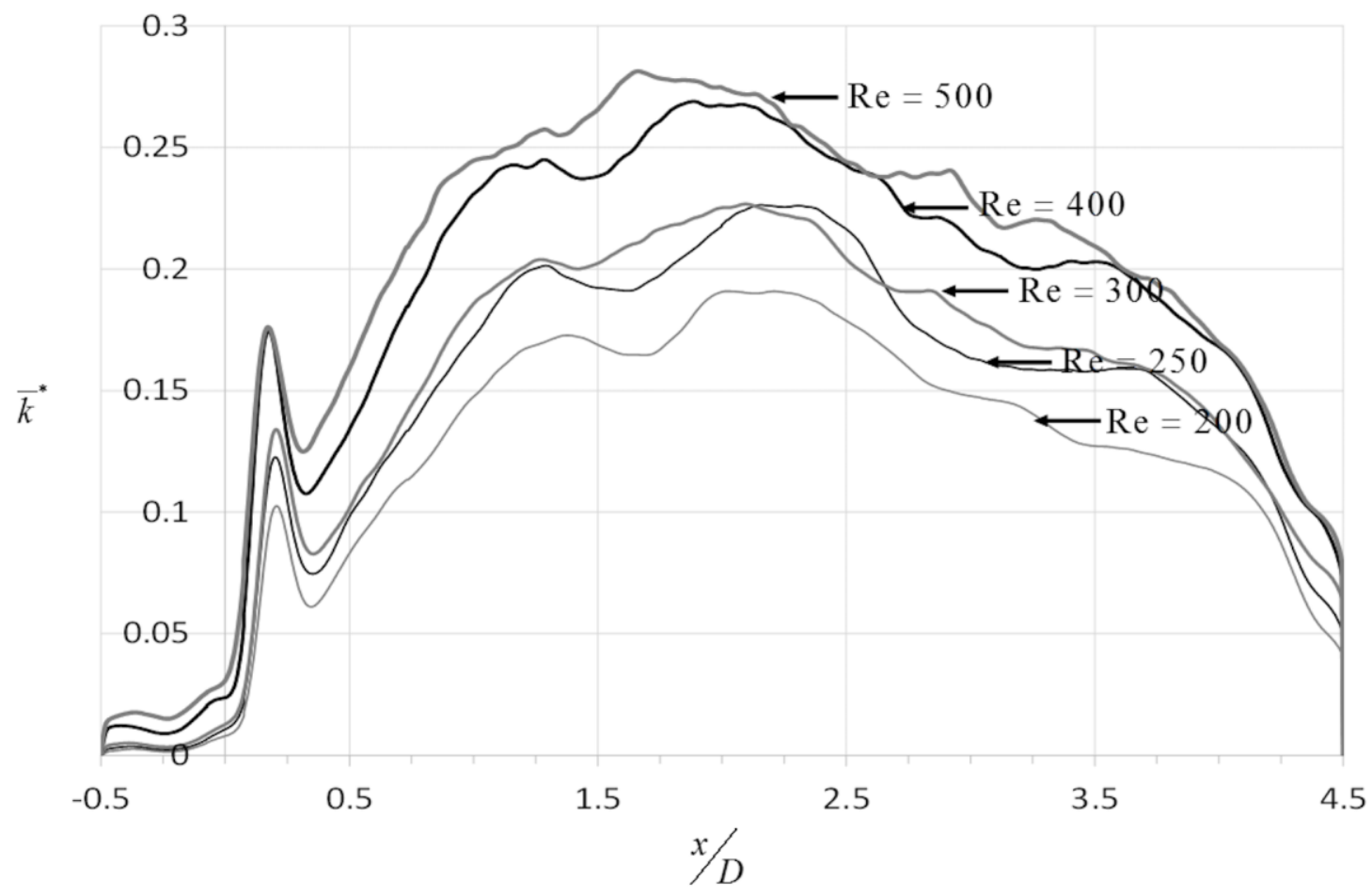

Figure 8. Normalized turbulent kinetic energy on y50 axis, for several Re, in CIJ 2D reactor. 


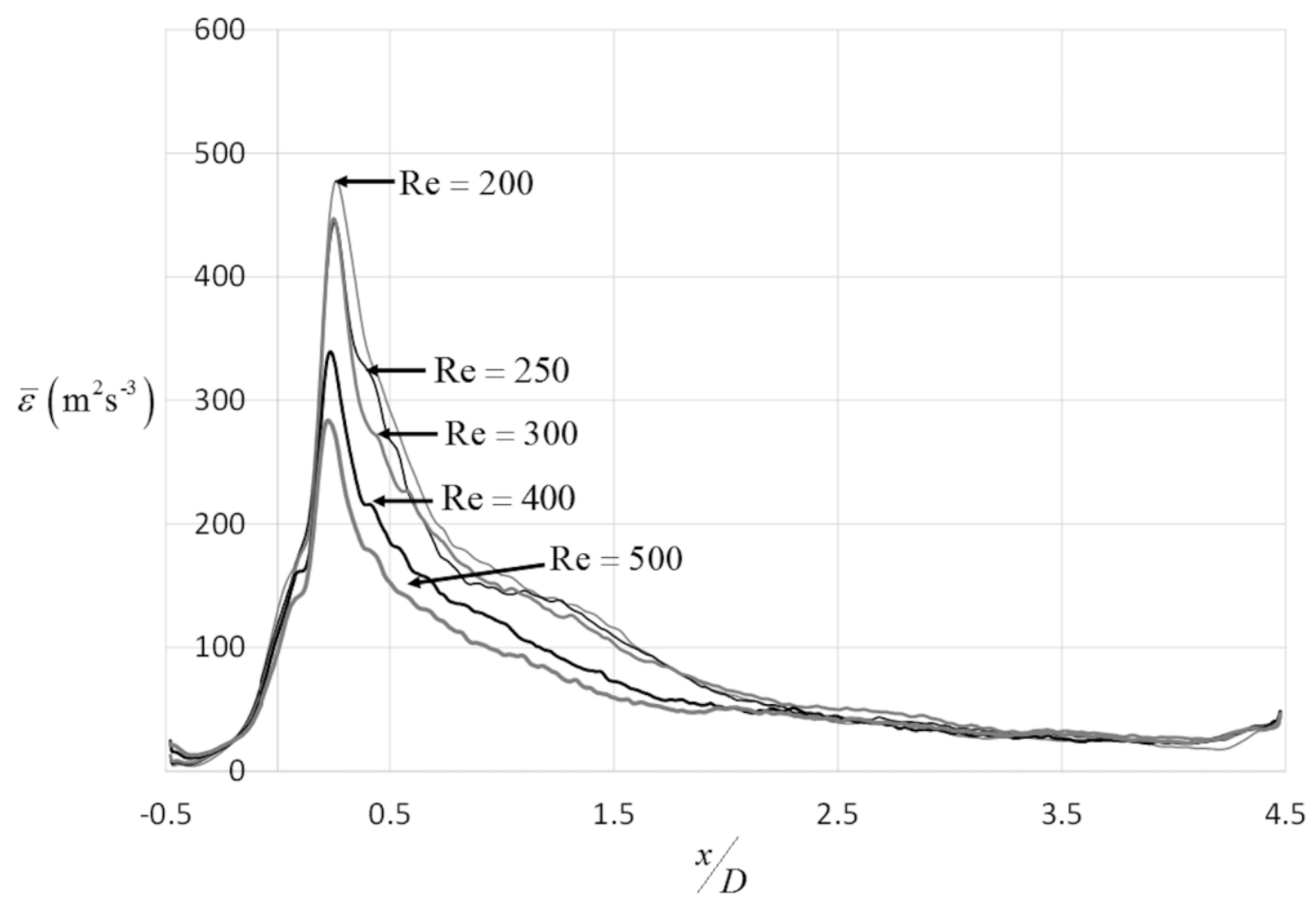

Figure 9. Turbulent kinetic energy dissipation rate on y50 axis, with several Re, in CIJ 2D reactor. 


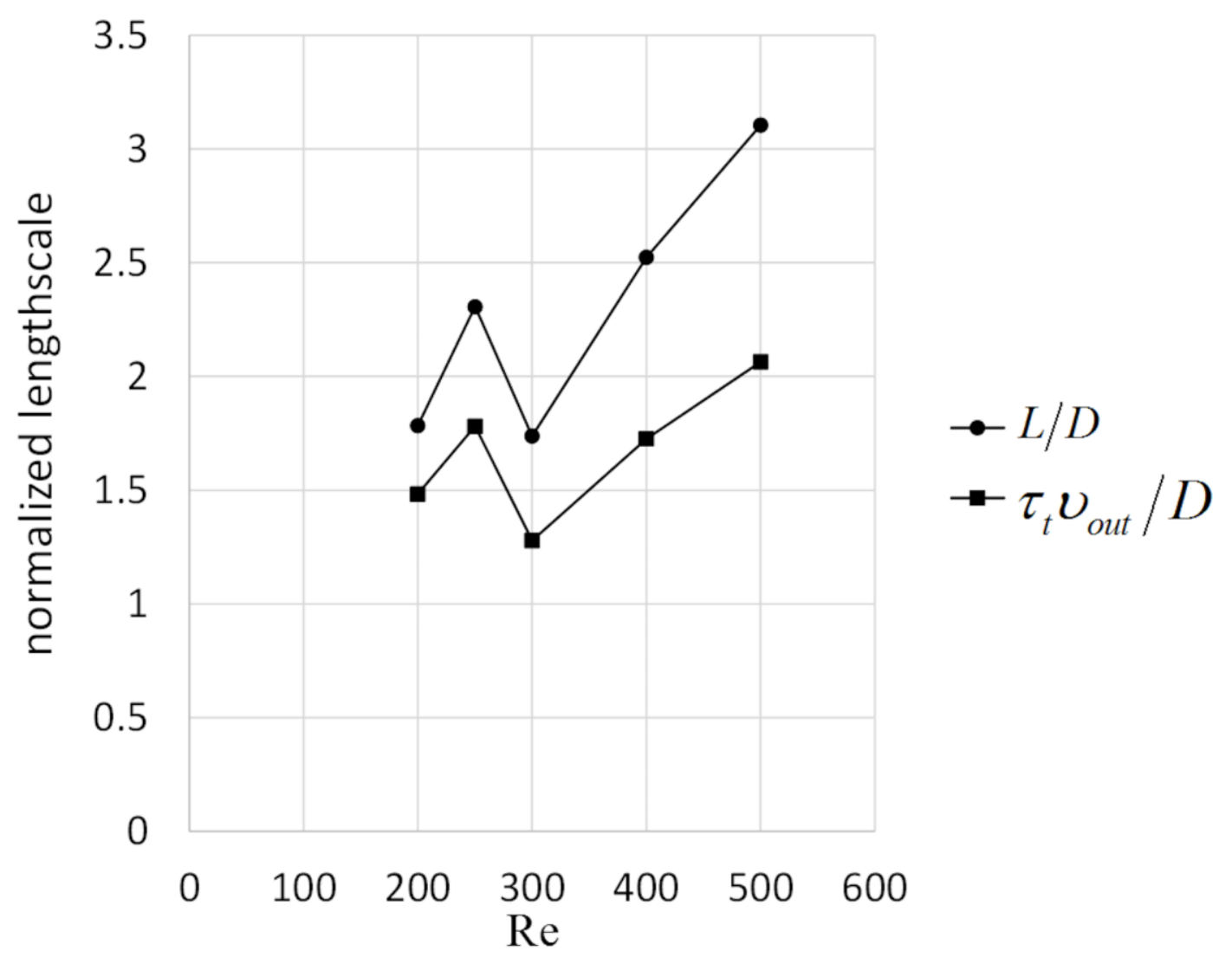

Figure 10. Normalized lengthscales L/D and T_t v_out /D on y50 axis, with several Re in the 2D CIJ. 


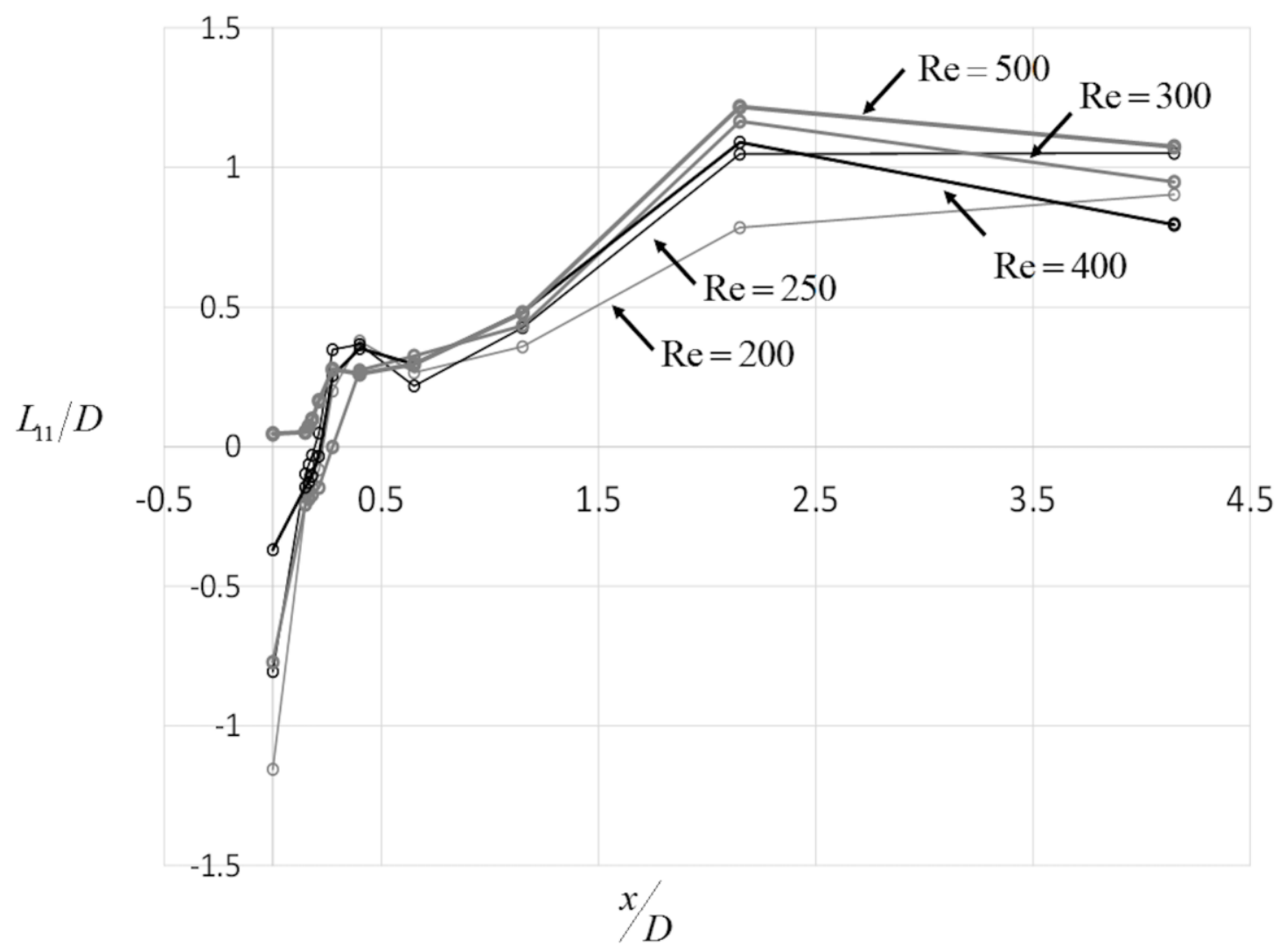

Figure 11. Normalized turbulence wavelength (L_11/D) obtained from 1000 time steps equally spaced through simulation along y50 axis. 


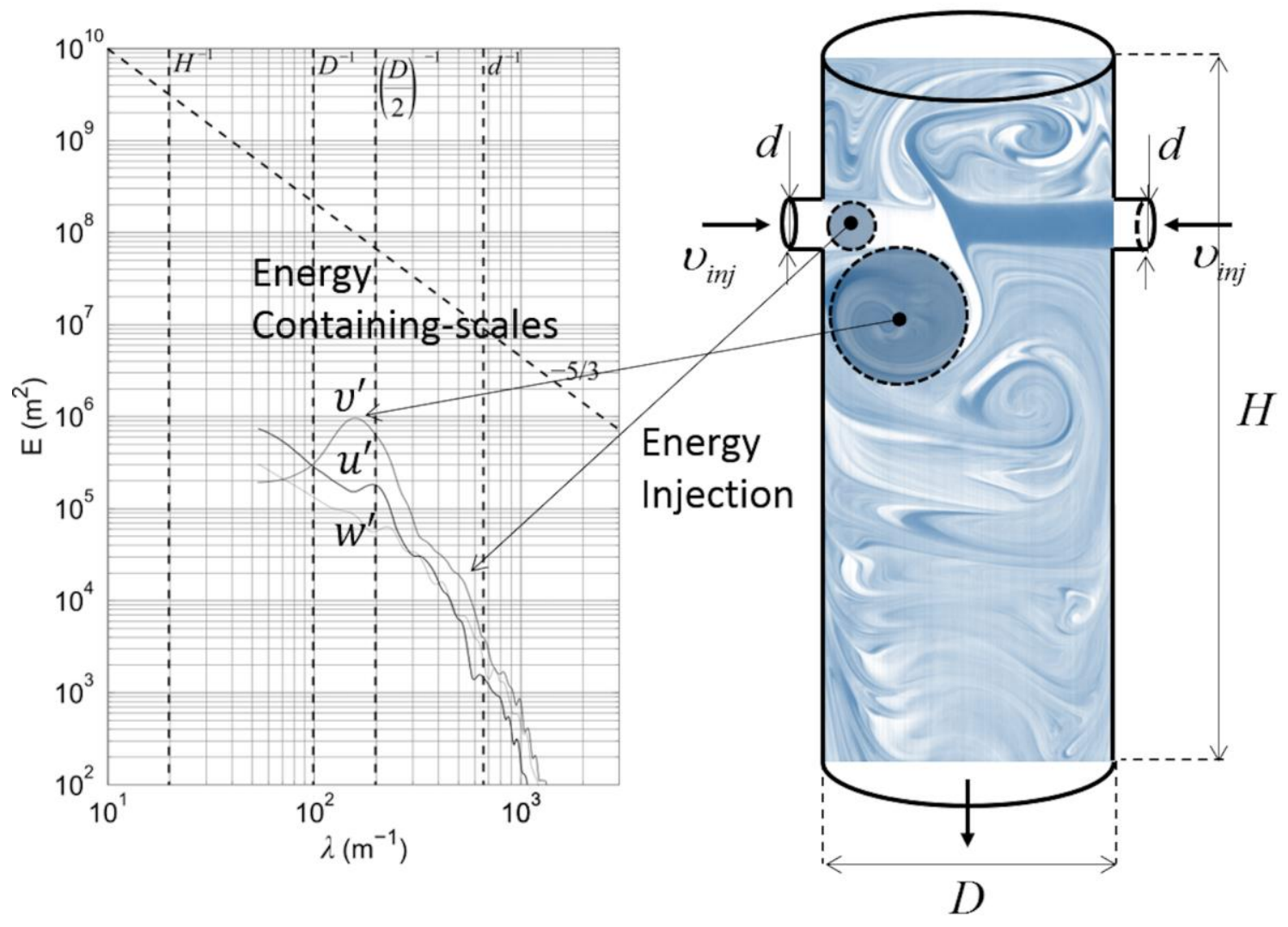

Figure 12. Power spectrum at $\mathrm{x} 5 \mathrm{~d} 1 \mathrm{D} / 2$ in CIJ 3D reactor for Re5150 numerical simulation, of u0 (black), v0 (dark grey), and w0 (light grey), and in CIJ 2D reactor for Re5300. 